\title{
REDSHIFTS, SAMPLE PURITY, AND BCG POSITIONS FOR THE GALAXY CLUSTER CATALOG FROM THE FIRST 720 SQUARE DEGREES OF THE SOUTH POLE TELESCOPE SURVEY
}

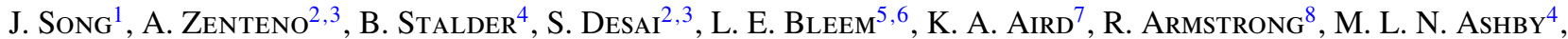 \\ M. Bayliss ${ }^{4,9}$, G. Bazin ${ }^{2,3}$, B. A. Benson ${ }^{5,10}$, E. Bertin ${ }^{11}$, M. Brodwin ${ }^{12}$, J. E. Carlstrom ${ }^{5,6,10,13,14}$, C. L. Chang ${ }^{5,10,14}$, \\ H. M. $\mathrm{ChO}^{15}$, A. Clocchiatti ${ }^{16}$, T. M. Crawford ${ }^{5,13}$, A. T. Crites $^{5,13}$, T. De HaAN ${ }^{17}$, M. A. Dobbs ${ }^{17}$, J. P. Dudley ${ }^{17}$, \\ R. J. Foley ${ }^{4}$, E. M. George ${ }^{18}$, D. Gettings ${ }^{19}$, M. D. Gladders ${ }^{5,13}$, A. H. Gonzalez ${ }^{19}$, N. W. Halverson ${ }^{20}$, \\ N. L. Harrington ${ }^{18}$, F. W. High ${ }^{5,13}$, G. P. Holder ${ }^{17}$, W. L. Holzapfel ${ }^{18}$, S. Hoover ${ }^{5,10}$, J. D. Hrubes ${ }^{7}$, M. JoY ${ }^{21}$,

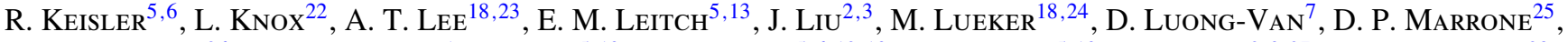 \\ M. McDonald ${ }^{26}$, J. J. McMahon ${ }^{1}$, J. Meht ${ }^{5,13}$, S. S. Meyer ${ }^{5,6,10,13}$, L. Mocand ${ }^{5,13}$, J. J. Mohr ${ }^{2,3,27}$, T. E. Montroy ${ }^{28}$, \\ T. Natoli ${ }^{5,6}$, D. Nurgaliev ${ }^{9}$, S. Padin ${ }^{5,13,24}$, T. PlagGe ${ }^{5,13}$, C. Pryke ${ }^{29}$, C. L. Reichardt ${ }^{18}$, A. Rest ${ }^{30}$, J. Ruel ${ }^{9}$, J. E. Ruhl ${ }^{28}$,

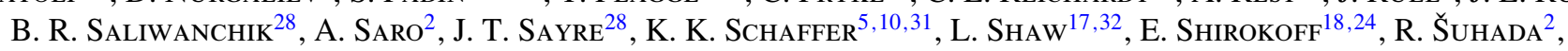 \\ H. G. Spieler ${ }^{23}$, S. A. Stanford ${ }^{22,33}$, Z. Staniszewsir ${ }^{28}$, A. A. Stark ${ }^{4}$, K. Story ${ }^{5,6}$, C. W. Stubbs ${ }^{4,9}$, A. VAn Engelen ${ }^{17}$, \\ K. VANDERLinde ${ }^{17}$, J. D. Vieira ${ }^{5,6,24}$, R. WilliamsON ${ }^{5,13}$, AND O. ZahN ${ }^{18,34}$ \\ ${ }^{1}$ Department of Physics, University of Michigan, 450 Church Street, Ann Arbor, MI 48109, USA \\ 2 Department of Physics, Ludwig-Maximilians-Universität, Scheinerstr. 1, D-81679 München, Germany \\ ${ }^{3}$ Excellence Cluster Universe, Boltzmannstr. 2, D-85748 Garching, Germany \\ ${ }^{4}$ Harvard-Smithsonian Center for Astrophysics, 60 Garden Street, Cambridge, MA 02138, USA \\ ${ }^{5}$ Kavli Institute for Cosmological Physics, University of Chicago, 5640 South Ellis Avenue, Chicago, IL 60637, USA \\ ${ }^{6}$ Department of Physics, University of Chicago, 5640 South Ellis Avenue, Chicago, IL 60637, USA \\ ${ }^{7}$ Department of Physics, University of Chicago, 5640 South Ellis Avenue, Chicago, IL 60637, USA \\ ${ }^{8}$ Department of Physics and Astronomy,University of Pennsylvania, Philadelphia, PA 19104, USA \\ ${ }^{9}$ Department of Physics, Harvard University, 17 Oxford Street, Cambridge, MA 02138, USA \\ ${ }^{10}$ Enrico Fermi Institute, University of Chicago, 5640 South Ellis Avenue, Chicago, IL 60637, USA \\ ${ }^{11}$ Institut d'Astrophysique de Paris, UMR 7095 CNRS, Université Pierre et Marie Curie, 98 bis boulevard Arago, F-75014 Paris, France \\ ${ }^{12}$ Department of Physics and Astronomy, University of Missouri, 5110 Rockhill Road, Kansas City, MO 64110, USA \\ ${ }^{13}$ Department of Astronomy and Astrophysics, University of Chicago, 5640 South Ellis Avenue, Chicago, IL 60637, USA \\ ${ }^{14}$ Argonne National Laboratory, 9700 South Cass Avenue, Argonne, IL 60439, USA \\ ${ }^{15}$ NIST Quantum Devices Group, 325 Broadway Mailcode 817.03, Boulder, CO 80305, USA \\ ${ }^{16}$ Departamento de Astronomia y Astrosifica, Pontificia Universidad Catolica, Santiago, Chile \\ ${ }^{17}$ Department of Physics, McGill University, 3600 Rue University, Montreal, Quebec H3A 2T8, Canada \\ ${ }^{18}$ Department of Physics, University of California, Berkeley, CA 94720, USA \\ ${ }^{19}$ Department of Astronomy, University of Florida, Gainesville, FL 32611, USA \\ ${ }^{20}$ Department of Astrophysical and Planetary Sciences and Department of Physics, University of Colorado, Boulder, CO 80309, USA \\ ${ }^{21}$ Department of Space Science, VP62, NASA Marshall Space Flight Center, Huntsville, AL 35812, USA \\ ${ }^{22}$ Department of Physics, University of California, One Shields Avenue, Davis, CA 95616, USA \\ ${ }^{23}$ Physics Division, Lawrence Berkeley National Laboratory, Berkeley, CA 94720, USA \\ ${ }^{24}$ Department of Astronomy, California Institute of Technology, 1200 East California Boulevard, Pasadena, CA 91125, USA \\ ${ }^{25}$ Steward Observatory, University of Arizona, 933 North Cherry Avenue, Tucson, AZ 85721, USA \\ ${ }^{26}$ Kavli Institute for Astrophysics and Space Research, Massachusetts Institute of Technology, 77 Massachusetts Avenue, Cambridge, MA 02139, USA \\ ${ }^{27}$ Max-Planck-Institut für Extraterrestrische Physik, Giessenbachstr., D-85748 Garching, Germany \\ ${ }^{28}$ Physics Department, Center for Education and Research in Cosmology and Astrophysics, Case Western Reserve University, Cleveland, OH 44106, USA \\ ${ }^{29}$ Physics Department, University of Minnesota, 116 Church Street S.E., Minneapolis, MN 55455, USA \\ ${ }^{30}$ Space Telescope Science Institute, 3700 San Martin Drive, Baltimore, MD 21218, USA \\ ${ }^{31}$ Liberal Arts Department, School of the Art Institute of Chicago, 112 South Michigan Avenue, Chicago, IL 60603, USA \\ 32 Department of Physics, Yale University, P.O. Box 208210, New Haven, CT 06520-8120, USA \\ ${ }^{33}$ Institute of Geophysics and Planetary Physics, Lawrence Livermore National Laboratory, Livermore, CA 94551, USA \\ ${ }^{34}$ Berkeley Center for Cosmological Physics, Department of Physics, University of California, and Lawrence Berkeley National Labs, Berkeley, CA 94720, USA \\ Received 2012 July 14; accepted 2012 October 16; published 2012 November 20
}

\begin{abstract}
We present the results of the ground- and space-based optical and near-infrared (NIR) follow-up of 224 galaxy cluster candidates detected with the Sunyaev-Zel'dovich (SZ) effect in the $720 \mathrm{deg}^{2}$ of the South Pole Telescope (SPT) survey completed in the 2008 and 2009 observing seasons. We use the optical/NIR data to establish whether each candidate is associated with an overdensity of galaxies and to estimate the cluster redshift. Most photometric redshifts are derived through a combination of three different cluster redshift estimators using red-sequence galaxies, resulting in an accuracy of $\Delta z /(1+z)=0.017$, determined through comparison with a subsample of 57 clusters for which we have spectroscopic redshifts. We successfully measure redshifts for 158 systems and present redshift lower limits for the remaining candidates. The redshift distribution of the confirmed clusters extends to $z=1.35$ with a median of $z_{\text {med }}=0.57$. Approximately $18 \%$ of the sample with measured redshifts lies at $z>0.8$. We estimate a lower limit to the purity of this SPT SZ-selected sample by assuming that all unconfirmed clusters are noise fluctuations in the SPT data. We show that the cumulative purity at detection significance $\xi>5(\xi>4.5)$ is $\geqslant 95 \%(\geqslant 70 \%)$. We present the red brightest cluster galaxy ( $\mathrm{rBCG})$ positions for the sample and examine the offsets between the SPT candidate position and the rBCG. The radial distribution of offsets is similar to that seen in
\end{abstract}


X-ray-selected cluster samples, providing no evidence that SZ-selected cluster samples include a different fraction of recent mergers from X-ray-selected cluster samples.

Key words: cosmology: observations - galaxies: clusters: general - galaxies: distances and redshifts

Online-only material: color figures

\section{INTRODUCTION}

In 2011 November, the South Pole Telescope (SPT; Carlstrom et al. 2011) collaboration completed a $2500 \mathrm{deg}^{2}$ survey, primarily aimed at detecting distant, massive galaxy clusters through their Sunyaev-Zel'dovich (SZ) effect signature. In Reichardt et al. (2012, R12 hereafter), the SPT team presented a catalog of 224 cluster candidates from $720 \mathrm{deg}^{2}$ observed in the 2008-2009 seasons. In this work, we present the optical and near-infrared (NIR) follow-up observations of the cluster candidates reported in R12, mainly focusing on follow-up strategy, confirmation, and empirical purity estimate for the cluster candidates, photometric redshift estimations of confirmed clusters, and the spatial position of the red brightest cluster galaxies (rBCGs).

Galaxy clusters have long been used for the study of structure formation and cosmology (e.g., Geller \& Beers 1982; White et al. 1993). Soon after the discovery of the cosmic acceleration (Schmidt et al. 1998; Perlmutter et al. 1999), it became clear that measurements of the redshift evolution of the cluster mass function could provide a powerful tool to further understand the underlying causes (Wang \& Steinhardt 1998; Haiman et al. 2001; Holder et al. 2001; Battye \& Weller 2003). More precise theoretical investigations (Majumdar \& Mohr 2003, 2004; Hu 2003; Molnar et al. 2004; Wang et al. 2004; Lima \& Hu 2005, 2007) identified the key challenges associated with cluster surveys, which include (1) producing large uncontaminated samples selected by an observable property that is closely related to the cluster mass, (2) measuring cluster redshifts for large samples, and (3) precisely calibrating the cluster masses.

Competitive approaches to producing large cluster samples include optical multiband surveys (e.g., Gladders \& Yee 2005; Koester et al. 2007), infrared surveys (e.g., Eisenhardt et al. 2008; Muzzin et al. 2008; Papovich 2008), X-ray surveys (e.g., Finoguenov et al. 2007, 2010; Pacaud et al. 2007; Vikhlinin et al. 2009; Mantz et al. 2010; Fassbender et al. 2011; LloydDavies et al. 2011; Šuhada et al. 2012), and millimeter-wave (mm-wave) surveys (Vanderlinde et al. 2010; Marriage et al. 2011; Planck Collaboration et al. 2011; Reichardt et al. 2012). The mm-wave surveys capitalize on the cluster SZ effect signature, which is produced by the inverse Compton scattering of cosmic microwave background photons by the energetic electrons within the cluster (Sunyaev \& Zel'dovich 1972). The surface brightness of the SZ effect is redshift independent, making SZ surveys a particularly powerful tool for identifying the most distant clusters. It is typical for X-ray and mm-wave surveys to have accompanying multiband optical imaging to enable photometric redshift measurements; these multiband optical data also enable a second stage of cluster candidate confirmation, verifying the purity estimation of the X-ray- or SZ-selected cluster samples.

Ideally, one would coordinate an SZ survey with a deep, multiband optical survey over the same region; indeed, the Dark
Energy Survey (DES ${ }^{35}$; Cease et al. 2008; Mohr et al. 2008) and the SPT are coordinated in this way. Because of the different development timelines for the two projects, it has been necessary to undertake extensive cluster-by-cluster imaging follow-up for SPT using a series of ground-based telescopes together with space-based NIR imaging (from Spitzer and WISE). The NIR data are of particular importance in the confirmation and redshift estimation of the $z>1$ massive galaxy clusters, which are especially interesting for both cosmological studies and studies of the evolution of clusters themselves. Pointed observations were used in High et al. (2010, H10 hereafter) to provide redshift and richness estimates of the SZ detections of Vanderlinde et al. (2010), and subsequently by Williamson et al. (2011) and Story et al. (2011).

Cluster samples from high-resolution SZ surveys can also be used to explore the evolution of cluster properties as a function of redshift. Previous studies using X-ray-selected clusters have identified a correlation between the dynamical state of a cluster and the projected offset between the X-ray centers and the BCG (e.g., Katayama et al. 2003; Sanderson et al. 2009; Haarsma et al. 2010; Fassbender et al. 2011; Stott et al. 2012). In principle, SZselected clusters can also serve as laboratories to search for this correlation, if the spatial resolution of SZ detections is high enough to detect the significance of offsets between the SZ centers and the BCGs. Systematic comparison between X-ray and SZ samples will indicate if the selection of the two methods differs in terms of the dynamical state of clusters.

This paper is structured as follows: We briefly describe the SPT data and methods for extracting the cluster sample in Section 2.1. In Section 2.2, we provide details of the followup strategy, as well as data processing. Section 3 is dedicated to a detailed description of the analysis of our follow-up data, including redshift estimation using optical and Spitzer data, the derivation of redshift lower limits for those systems that are not confirmed, and the selection of rBCGs in the clusters. Results are presented in Section 4 and discussed further in Section 5. Throughout this paper, we use the $A B$ magnitude system for optical and NIR observations unless otherwise noted in the text.

\section{DISCOVERY AND FOLLOW-UP}

\subsection{SPT Data}

Here we briefly summarize the analysis of the SPT data and the extraction of cluster candidates from that data; we refer the reader to R12 and previous SPT cluster publications (Staniszewski et al. 2009; Vanderlinde et al. 2010; Williamson et al. 2011) for more details.

The SPT operates in three frequency bands, although only data from the $95 \mathrm{GHz}$ and $150 \mathrm{GHz}$ detectors were used in finding clusters. The data from all detectors at a given observing frequency during an observing period (usually 1-2 hr) are

\footnotetext{
35 http://www.darkenergysurvey.org
} 
Table 1

Optical and Infrared Imagers

\begin{tabular}{|c|c|c|c|c|c|c|c|}
\hline Ref. $^{a}$ & Site & Telescope & $\begin{array}{l}\text { Aperture } \\
\text { (m) }\end{array}$ & Camera & Filters $^{\mathrm{b}}$ & Field & $\begin{array}{c}\text { Pixel Scale } \\
\text { (") }\end{array}$ \\
\hline 1 & Cerro Tololo & Blanco & 4 & MOSAIC-II & griz & $36^{\prime} \times 36^{\prime}$ & 0.27 \\
\hline 3 & Las Campanas & Magellan/Clay & 6.5 & LDSS3 & griz & 8.3 diam. circle & 0.189 \\
\hline $4^{c}$ & Las Campanas & Magellan/Clay & 6.5 & Megacam & $g r i$ & $25^{\prime} \times 25^{\prime}$ & 0.16 \\
\hline 5 & Las Campanas & Swope & 1 & SITe3 & $B V R I$ & $14^{\prime} .8 \times 22^{\prime} .8$ & 0.435 \\
\hline 7 & $\ldots$ & Spitzer Space Telescope & 0.85 & IRAC & $3.6 \mu \mathrm{m}, 4.5 \mu \mathrm{m}$ & $5.2 \times 5.2$ & 1.2 \\
\hline 8 & $\ldots$ & WISE & 0.40 & $\ldots$ & $3.4 \mu \mathrm{m}, 4.6 \mu \mathrm{m}$ & $47^{\prime} \times 47^{\prime}$ & 6 \\
\hline
\end{tabular}

Notes. Optical and infrared cameras used in SPT follow-up observations.

a Shorthand alias used in Table 3.

b Not all filters were used on every cluster.

${ }^{\mathrm{c}}$ Megacam data were acquired for a large follow-up weak-lensing program.

combined into a single map. The data undergo quality cuts and are high-pass filtered and inverse-noise weighted before being combined into a map. Many individual-observation maps of every field are co-added (again using inverse noise weighting) into a full-depth map of that field, and the individual-observation maps are differenced to estimate the map noise. The $95 \mathrm{GHz}$ and $150 \mathrm{GHz}$ full-depth maps of a given field are then combined using a spatial-spectral matched filter (e.g., Melin et al. 2006) that optimizes signal to noise on cluster-shaped objects with an SZ spectral signature. Cluster candidates are identified in the resulting filtered map using a simple peak detection algorithm, and each candidate is assigned a signal-to-noise value based on the peak amplitude divided by the rms of the filtered map in the neighborhood of the peak. Twelve different matched filters are used, each assuming a different scale radius for the cluster, and the maximum signal to noise for a given candidate across all filter scales is referred to as $\xi$, which we use as our primary SZ observable. In 2008, the $95 \mathrm{GHz}$ detectors in the SPT receiver had significantly lower sensitivity than the $150 \mathrm{GHz}$ array, and the cluster candidates from those observations are identified using $150 \mathrm{GHz}$ data only; the candidates from 2009 observations were identified using data from both bands. The data from the two observing seasons yielded a total of 224 cluster candidates with $\xi \geqslant 4.5$ - the sample discussed here.

\subsection{Optical/NIR Imaging}

The cluster candidates detected using the method described above are followed up by optical and, in many cases, NIR instruments. In this section, we describe the overall optical/NIR follow-up strategy, the different imaging and spectroscopic observations and facilities used, and the data reduction methods used to process the raw images to catalogs.

\subsubsection{Imaging Observations}

The optical/NIR follow-up strategy has evolved since the first SPT-SZ candidates were identified. Originally we imaged regions of the sky with uniformly deep, multiband observations in griz optical bands to confirm SZ detections and estimate redshifts as in Staniszewski et al. (2009). For the first SPT cluster candidates, we used imaging from the Blanco Cosmology Survey (BCS; Desai et al. 2012) to follow up candidates in parts of the 2008 fields. The BCS is a 60 night, $\sim 80 \mathrm{deg}^{2}$ NOAO survey program carried out in 2005-2008 using the Blanco/MOSAIC2 griz filters. The BCS survey was completed to the required depths for $5 \sigma$ detection at $0.4 L^{*}$ within 2 .".3 apertures up to $z \sim 1$. The goal of this survey was to provide optical imaging over a limited area of the SPT survey to enable rapid optical follow-up of the initial SPT survey fields.

For clusters outside the BCS region, we initially obtained deep griz imaging on a cluster-by-cluster basis. But as the SPT survey proceeded and the cluster candidate list grew, it became clear that this strategy was too costly, given the limited access to follow-up time. Moreover, eventually the full SPT region will be imaged to uniform $10 \sigma$ depths of mag $\sim 24$ in griz by the DES. We therefore switched to an adaptive strategy of follow-up in which we observed each SPT cluster candidate to the depth required to find an optical counterpart and determine its redshift.

For each SPT cluster candidate, we perform an initial prescreening of candidates using the Digitized Sky Survey (DSS). ${ }^{36}$ We examine DSS images using three bands ${ }^{37}$ for each cluster candidate to determine whether it is "low- $z$ " or "high- $z$," where the redshift boundary lies roughly at $z=0.5$. We find that this visual classification identifies spectroscopically and photometrically confirmed SPT clusters out to $z=0.5$ in the DSS photographic plates. We use the DSS designation to prioritize the target list for the appropriate telescope, instrument, and filters with which we observe each candidate. Specifically, candidates that are clearly identified in DSS images are likely to be low- $z$ clusters and are designated for follow-up observations on small-aperture (1-2 m) telescopes. Otherwise, candidates are classified as high $z$ candidates and therefore designated for large-aperture $(4-6.5 \mathrm{~m})$ telescopes. The various ground- and space-based facilities used to collect optical/NIR imaging data on SPT clusters are summarized in Table 1. Each telescope/ instrument combination is assigned a numeric alias that is used to identify the source of the redshift data for each cluster in Table 3.

For the $\geqslant 4 \mathrm{~m}$ class observations, we use an adaptive filter and exposure time strategy so that we can efficiently bracket the cluster member galaxy's $4000 \AA$ Areak to the depth required for redshift estimation. In this approach we start with a first imaging pass, where each candidate is observed in the $g, r$, and $z$ bands to achieve a depth corresponding to a $5 \sigma$ detection of a $0.4 L^{*}$ galaxy at $z=0.8, \sim 23.5 \mathrm{mag}$ and $21.8 \mathrm{mag}$ in $r$ and $z$ bands, respectively, based on the Bruzual \& Charlot (2003) red-sequence model (for more details about the model,

\footnotetext{
$36 \mathrm{http} / / /$ archive.stsci.edu/dss/

37 http://gsss.stsci.edu/SkySurveys/Surveys.htm
} 
see Section 3.1). Observations are also taken in a single blue filter for photometric calibration using the stellar locus (discussed in Section 2.2.2). For candidates with no obvious optical counterpart after first-pass observations, a second pass is executed to get to $z=0.9, \sim 23 \mathrm{mag}$ and $22 \mathrm{mag}$ in $i$ and $z$ bands, respectively.

If the candidate is still not confirmed after the second pass in $i$ and $z$ bands, and is not covered by the Spitzer/IRAC pointed observations described below, we attempt to obtain groundbased NIR imaging for that candidate using the NEWFIRM camera on the CTIO Blanco telescope. The data presented here are imaged with NEWFIRM during three observing runs in 2010 and 2011, yielding $K_{s}$ data for a total of 31 candidates. Typical observations in $K_{s}$ consist of 16 point dither patterns, with $60 \mathrm{~s}$ exposures divided among 6 co-adds at each dither position. Median seeing during the 2010 runs was $1^{\prime \prime}$. 05 ; during the 2011 run, observing conditions were highly variable and the seeing ranged from $1^{\prime \prime} .05$ to 2 .' 6 with median seeing $\sim 1^{\prime \prime} .2$.

We note that most of the galaxy cluster candidates in this work with significance $\xi>4.8$ were imaged with Spitzer (Werner et al. 2004). More specifically, Spitzer/IRAC (Fazio et al. 2004) imaging has been obtained for 99 SZ cluster candidates in this work. The on-target Spitzer observations consist of $8 \times 100 \mathrm{~s}$ and $6 \times 30 \mathrm{~s}$ dithered exposures at $3.6 \mu \mathrm{m}$ and $4.5 \mu \mathrm{m}$, respectively. The deep $3.6 \mu \mathrm{m}$ observations should produce $5 \sigma$ detections of passively evolving $0.1 L^{*}$ cluster galaxies at $z=1.5(\sim 17.8 \mathrm{mag}$ (Vega) at $z=1.5$ ).

For some of the NIR analysis, we augment the data from our Spitzer and NEWFIRM observations with the recently released all-sky Wide-field Infrared Survey Explorer (WISE; Wright et al. 2010) data. Finally, we note that a few of the clusters were observed with Magellan/Megacam to obtain weak gravitational lensing mass measurements (High et al. 2012). These data are naturally much deeper than our initial follow-up imaging.

\subsubsection{Data Processing}

We use two independent optical data processing systems. One system, which we refer to as the PHOTPIPE pipeline, is used to process all optical data except Magellan/Megacam data, and the other, which is a development version of the Dark Energy Survey data management (DESDM) system, is used only to process the Blanco/Mosaic2 data. PHOTPIPE was used to process optical data for previous SPT cluster catalogs (Vanderlinde et al. 2010; High et al. 2010; Williamson et al. 2011); the DESDM system has been used as a cross-check in these works and was the primary reduction pipeline used in Staniszewski et al. (2009).

The basic stages of the PHOTPIPE pipeline, initially developed for the SuperMACHO and ESSENCE projects and described in Rest et al. (2005), Garg et al. (2007), and Miknaitis et al. (2007), include flat-fielding, astrometry, coadding, and source extraction. Further details are given in H10. In the DESDM system (Ngeow et al. 2006; Mohr et al. 2008; Desai et al. 2012), the data from each night first undergo detrending corrections, which include cross-talk correction, overscan correction, trimming, bias subtraction, flat fielding, and illumination correction. Single-epoch images are not remapped to avoid correlating noise, and so we also perform a pixel-scale correction that brings all sources on an image to a common photometric zero point. For $i$ and $z$ bands we also carry out a fringe correction. Astrometric calibration is done by using the AstrOmatic code SCAMP (Bertin 2006) and the USNO-B catalog. Color terms to transform to the Sloan Digital Sky Survey (SDSS) system rely on photometric solutions derived from ob- servations of SDSS equatorial fields during photometric nights (Desai et al. 2012).

In both pipelines, co-addition is done using SWarp (Bertin et al. 2002). In the DESDM system the single epoch images contributing to the co-add are brought to a common zero point using stellar sources common to pairs of images. The final photometric calibration of the co-add images is carried out using the stellar color-color locus as a constraint on the zeropoint offsets between neighboring bands (e.g., High et al. 2009), where the absolute photometric calibration comes from the Two Micron All Sky Survey (2MASS; Skrutskie et al. 2006). For griz photometry the calibration is done with reference to the median SDSS stellar locus (Covey et al. 2007), but for the Swope data using Johnson filters, the calibration relies on a stellar locus derived from a sequence of models of stellar atmospheres from PHOENIX (Brott \& Hauschildt 2005) with empirically measured CCD, filter, and atmosphere responses. Cataloging is done using SExtractor (Bertin \& Arnouts 1996), and within the DESDM catalogs we calibrate mag_auto using stellar locus.

Quality checks of the photometry are carried out on a clusterby-cluster basis using the scatter of stars about the expected stellar locus and the distribution of offsets in the single-epoch photometry as a function of calibrated magnitude (so-called photometric repeatability tests). Poor-quality data or failed calibrations are easily identified as those co-adds with high stellar locus scatter and or high scatter in the photometric repeatability tests (see Desai et al. 2012).

NEWFIRM imaging data are reduced using the FATBOY pipeline (Eikenberry et al. 2006), originally developed for the FLAMINGOS-2 instrument, and modified to work with NEWFIRM data in support of the Infrared Bootes Imaging Survey (Gonzalez et al. 2010). Individual processed frames are combined using SCAMP and SWarp, and photometry is calibrated to 2 MASS.

Spitzer/IRAC data are reduced following the method of Ashby et al. (2009). Briefly, we correct for column pulldown, mosaic the individual exposures, resample to 0.86 pixels (half the solid angle of the native IRAC pixels), and reject cosmic rays. Magnitudes are measured in $4^{\prime \prime}$ diameter apertures and corrected for the $38 \%$ and $40 \%$ loss at $3.6 \mu \mathrm{m}$ and $4.5 \mu \mathrm{m}$, respectively, due to the broad point-spread function (PSF; see Table 3 in Ashby et al. 2009). The Spitzer photometry is crucial to the measurement of photometric redshifts for clusters at $z \gtrsim 0.8$, as described in Section 3.1.

The acquisition and processing for the initial weak lensing Megacam data is described in detail in High et al. (2012). These data are reduced separately from the other imaging data using the Smithsonian Astrophysical Observatory (SAO) Megacam reduction pipeline. Standard raw CCD image processing, cosmic-ray removal, and flat-fielding are performed, as well as an additional illumination correction to account for a low-order scattered light pattern. The final images are co-added onto a single pixel grid with a pixel scale of 0'.16 using SWarp. Sources are detected in the co-added data in dual-image mode using SExtractor, where the $r$-band image serves as the detection image. The photometry is calibrated by fitting colors to the stellar locus, and color-term corrections are accounted for in this step. The color term is roughly $0.10(g-i)$ for the $g^{\prime}$ band, $-0.02(g-i)$ for the $r^{\prime}$ band, and $-0.03(g-i)$ for the $i^{\prime}$ band.

\subsection{Spectroscopic Observations}

We have targeted many of the SPT clusters with long-slit and multi-object spectroscopy, and some of the spectroscopic 
Table 2

Spectroscopic Follow-up

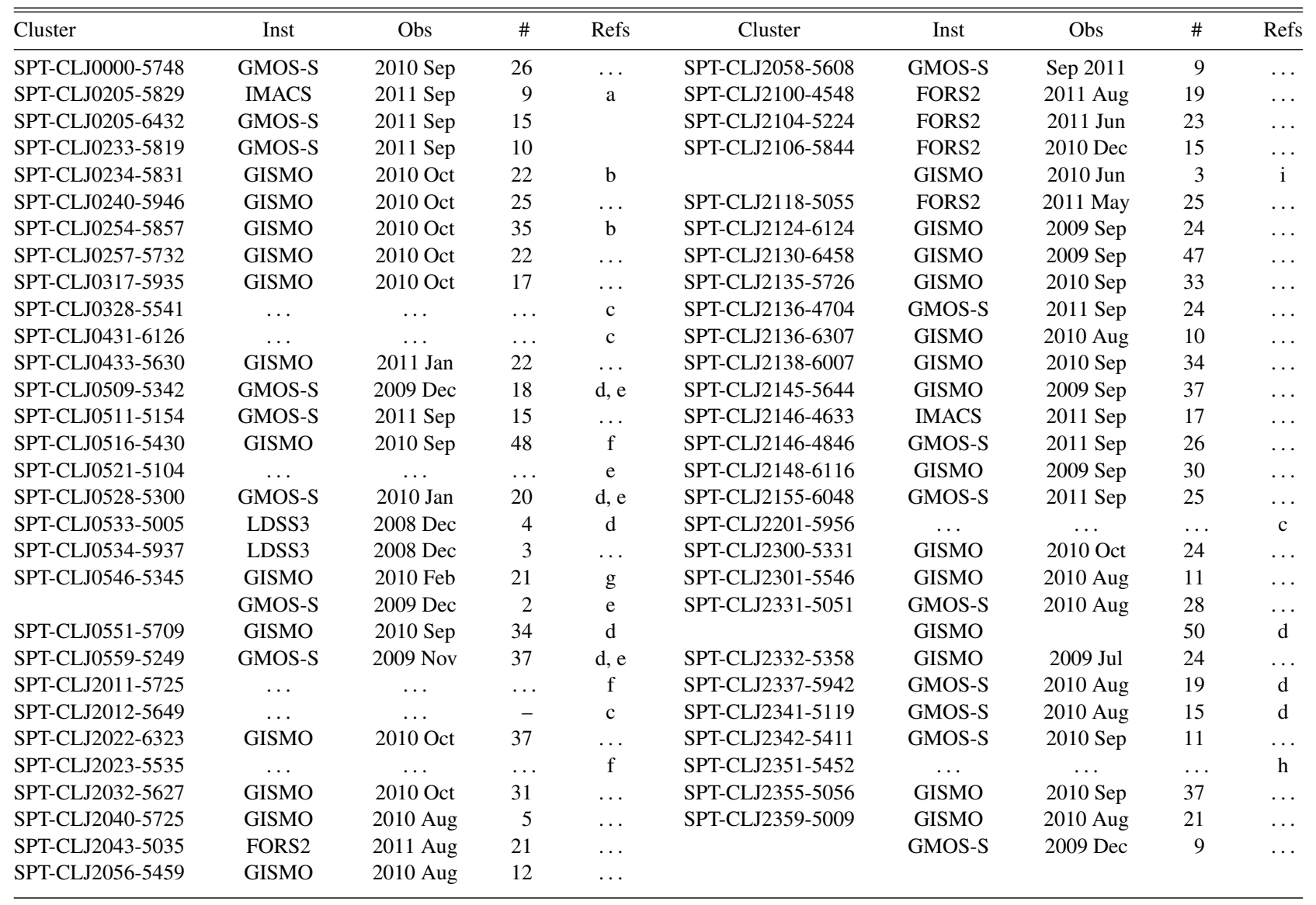

Notes. Instruments (Inst): GMOS-S on Gemini South $8 \mathrm{~m}$, IMACS on Magellan Baade 6.5 m, GISMO complement to IMACS on Magellan Baade $6.5 \mathrm{~m}$, LDSS3 on Magellan Clay 6.5, FORS2 on VLT Antu $8 \mathrm{~m}$. Observing dates (Obs): dates each data taken. Number of galaxies (\#): number of galaxies used in deriving redshifts. References (Refs): ${ }^{\mathrm{a}}$ Stalder et al. (2012), ${ }^{\mathrm{b}}$ Williamson et al. (2011), ${ }^{\mathrm{c}}$ Struble \& Rood (1999), ${ }^{\mathrm{d}}$ High et al. (2010), ${ }^{\mathrm{e}}$ Sifon et al. (2012), ${ }^{\mathrm{f}}$ Böhringer et al. (2004), ${ }^{\mathrm{g}}$ Brodwin et al. (2010), ${ }^{\mathrm{h}}$ Buckley-Geer et al. (2011), ${ }^{\mathrm{i}}$ Foley et al. (2011).

redshifts have appeared in previous SPT publications. We have used a variety of instruments: GMOS-S ${ }^{38}$ on Gemini South, FORS2 (Appenzeller et al. 1998) on Very Large Telescope (VLT), LDSS3 on Magellan-Clay, and the IMACS camera on Magellan Baade (in long-slit mode and with the GISMO ${ }^{39}$ complement).

A detailed description of the configurations, observing runs, and reductions will be presented elsewhere (J. Ruel et al., in preparation). For a given cluster we target bright galaxies that lie on the clusters' red sequence and observe these galaxies with a combination of filter and disperser that yields a lowresolution spectrum around their Ca II H\&K lines and $4000 \AA$ break. CCD reductions are made using standard packages, including COSMOS (Kelson 2003) for IMACS data and IRAF ${ }^{40}$ for GMOS and FORS2. Redshift measurements are made by cross-correlation with the RVSAO package (Kurtz \& Mink 1998) and a proprietary template fitting method that uses SDSS DR2 templates. Results are then visually confirmed using strong spectral features.

In Table 2, the source for every spectroscopic redshift is listed, along with the number of cluster members used in

\footnotetext{
38 http://www.gemini.edu/node/10625

$39 \mathrm{http} / / / \mathrm{www} .1 \mathrm{co.cl} /$ telescopes-information/magellan/instruments/imacs/ gismo/gismoquickmanual.pdf

40 http://iraf.noao.edu
}

deriving the redshift. For clusters for which we report our own spectroscopic measurements, we list an instrument name and observation date; we give a literature reference for those for which we report a value from the literature. In Table 3, we report spectroscopic redshifts for 57 clusters, of which 36 had no previous spectroscopic redshift in the literature. Unless otherwise noted, the reported cluster redshift is the robust biweight average of the redshifts of all spectroscopically confirmed cluster members, and the cluster redshift uncertainty is found from bootstrap resampling.

\section{METHODOLOGY}

In this section, we describe the analysis methods used to (1) extract cluster redshift estimates and place redshift limits, (2) empirically verify the estimates of catalog purity, and (3) measure rBCG positions.

\subsection{Photometric Redshifts}

Using the procedure described in Section 2.2, we obtain ground-based imaging data and galaxy catalogs that in most cases allow us to identify an obvious overdensity of redsequence galaxies within approximately an arcminute of the SPT candidate position. For these optically confirmed cluster candidates, we proceed to estimate a photometric redshift. 
Table 3

All Candidates above $\xi=4.5$ in $720 \mathrm{deg}^{2}$ of the SPT-SZ Survey

\begin{tabular}{|c|c|c|c|c|c|c|c|c|c|c|c|c|}
\hline \multirow[t]{2}{*}{ SPT ID } & \multicolumn{2}{|c|}{ Position } & \multirow[t]{2}{*}{$\xi$} & \multirow[t]{2}{*}{$z_{\text {spec }}{ }^{\mathrm{a}}$} & \multirow{2}{*}{$\begin{array}{c}z_{\text {comb }} \pm \sigma_{z_{\text {comb }}} \\
\text { (or redshift lower limit) }\end{array}$} & \multirow[t]{2}{*}{ Flag $^{b}$} & \multicolumn{3}{|c|}{ NIR Blank Field Probability $P_{\text {blank }}(\%)^{\mathrm{c}}$} & \multicolumn{2}{|c|}{ rBCG Position } & \multirow[t]{2}{*}{ Imaging Ref. ${ }^{\mathrm{d}}$} \\
\hline & R.A. (deg) & Decl. (deg) & & & & & NEWFIRM & Spitzer & WISE & R.A. (deg) & Decl. (deg) & \\
\hline SPT-CLJ0000-5748 & 0.2496 & -57.8066 & 5.48 & 0.702 & $0.68 \pm 0.03$ & 1 & . & $\ldots$ & $\ldots$ & 0.2503 & -57.8093 & 1,2 \\
\hline SPT-CLJ0201-6051 & 30.3933 & -60.8592 & 4.83 & $\ldots$ & $>1.0$ & $\ldots$ & 61.3 & $\ldots$ & 100.0 & $\ldots$ & $\ldots$ & 1 \\
\hline SPT-CLJ0203-5651 ${ }^{\mathrm{e}}$ & 30.8309 & -56.8612 & 4.98 & $\ldots$ & $>1.0$ & $\ldots$ & 28.1 & $\ldots$ & 56.5 & $\ldots$ & $\ldots$ & 1 \\
\hline SPT-CLJ0205-5829 & 31.4437 & -58.4855 & 10.54 & 1.322 & $1.30 \pm 0.07$ & 3 & 8.0 & 2.0 & 1.8 & 31.4510 & -58.4803 & 1 \\
\hline SPT-CLJ0205-6432 & 31.2786 & -64.5461 & 6.02 & 0.744 & $0.75 \pm 0.03$ & 1 & 6.8 & 0.5 & 17.3 & 31.3244 & -64.5583 & 1 \\
\hline SPT-CLJ0209-5452 & 32.3491 & -54.8794 & 4.52 & $\ldots$ & $0.42 \pm 0.03$ & 1 & $\ldots$ & $\ldots$ & $\ldots$ & 32.3494 & -54.8720 & 1 \\
\hline SPT-CLJ0211-5712 & 32.8232 & -57.2157 & 4.77 & $\ldots$ & $>1.0$ & $\ldots$ & 26.7 & $\ldots$ & 56.1 & $\ldots$ & $\ldots$ & 1 \\
\hline SPT-CLJ0216-5730 & 34.1363 & -57.5100 & 4.72 & $\ldots$ & $>1.0$ & $\ldots$ & 43.4 & $\ldots$ & 80.6 & $\ldots$ & $\ldots$ & 1,2 \\
\hline SPT-CLJ0216-6409 & 34.1723 & -64.1562 & 5.54 & $\ldots$ & $0.64 \pm 0.03$ & 1 & $\ldots$ & $\ldots$ & $\ldots$ & 34.1599 & -64.1599 & 1 \\
\hline SPT-CLJ0218-5826 & 34.6251 & -58.4386 & 4.54 & $\ldots$ & $0.57 \pm 0.03$ & 1 & & $\ldots$ & $\ldots$ & 34.6267 & -58.4421 & 1,2 \\
\hline SPT-CLJ0221-6212 & 35.4382 & -62.2044 & 4.71 & $\ldots$ & $>1.2$ & $\ldots$ & 75.7 & $\ldots$ & 84.9 & $\ldots$ & & 1 \\
\hline SPT-CLJ0230-6028 & 37.6410 & -60.4694 & 5.88 & $\ldots$ & $0.74 \pm 0.08$ & 3 & $\ldots$ & 11.3 & 0.3 & 37.6354 & -60.4628 & 3 \\
\hline SPT-CLJ0233-5819 & 38.2561 & -58.3269 & 6.64 & 0.663 & $0.76 \pm 0.07$ & 3 & 4.2 & 0.0 & $\ldots$ & 38.2541 & -58.3269 & 1 \\
\hline SPT-CLJ0234-5831 & 38.6790 & -58.5217 & 14.65 & 0.415 & $0.42 \pm 0.03$ & 1 & $\ldots$ & $\ldots$ & $\ldots$ & 38.6762 & -58.5236 & 1 \\
\hline SPT-CLJ0239-6148 & 39.9120 & -61.8032 & 4.67 & $\ldots$ & $>1.1$ & $\ldots$ & 44.2 & $\ldots$ & 38.0 & $\cdots$ & $\ldots$ & 1,2 \\
\hline SPT-CLJ0240-5946 & 40.1620 & -59.7703 & 9.04 & 0.400 & $0.41 \pm 0.03$ & 1 & $\ldots$ & $\ldots$ & $\ldots$ & 40.1599 & -59.7635 & 3 \\
\hline SPT-CLJ0240-5952 & 40.1982 & -59.8785 & 4.65 & $\ldots$ & $0.62 \pm 0.03$ & 3 & $\ldots$ & $\ldots$ & $\ldots$ & 40.2048 & -59.8732 & 5 \\
\hline SPT-CLJ0242-6039 & 40.6551 & -60.6526 & 4.92 & $\ldots$ & $>1.5$ & $\ldots$ & $\ldots$ & 51.5 & 64.9 & . & & 2 \\
\hline SPT-CLJ0243-5930 & 40.8616 & -59.5132 & 7.42 & $\ldots$ & $0.65 \pm 0.03$ & 1 & $\ldots$ & $\ldots$ & $\ldots$ & 40.8628 & -59.5172 & 3 \\
\hline SPT-CLJ0249-5658 & 42.4068 & -56.9764 & 5.44 & $\ldots$ & $0.23 \pm 0.02$ & 1 & $\ldots$ & $\ldots$ & $\ldots$ & 42.3918 & -56.9870 & 1 \\
\hline SPT-CLJ0253-6046 & 43.4605 & -60.7744 & 4.83 & $\ldots$ & $0.44 \pm 0.02$ & 1 & $\ldots$ & $\ldots$ & $\ldots$ & 43.4508 & -60.7499 & 1 \\
\hline SPT-CLJ0254-5857 & 43.5729 & -58.9526 & 14.42 & 0.438 & $0.43 \pm 0.03$ & 1 & $\ldots$ & $\ldots$ & $\ldots$ & 43.5365 & -58.9718 & 3 \\
\hline SPT-CLJ0254-6051 & 43.6015 & -60.8643 & 6.71 & $\ldots$ & $0.44 \pm 0.02$ & 1 & $\ldots$ & $\ldots$ & $\ldots$ & 43.5884 & -60.8689 & 1 \\
\hline SPT-CLJ0256-5617 & 44.1009 & -56.2973 & 7.54 & $\ldots$ & $0.63 \pm 0.03$ & 1 & $\ldots$ & $\ldots$ & $\ldots$ & 44.0880 & -56.3031 & 3 \\
\hline SPT-CLJ0257-5732 & 44.3516 & -57.5423 & 5.40 & 0.434 & $0.42 \pm 0.02$ & 1 & $\ldots$ & $\ldots$ & $\ldots$ & 44.3373 & -57.5484 & 1 \\
\hline SPT-CLJ0257-5842 & 44.3924 & -58.7117 & 5.38 & $\ldots$ & $0.42 \pm 0.02$ & 1 & $\ldots$ & $\ldots$ & $\ldots$ & 44.4374 & -58.7045 & 1 \\
\hline SPT-CLJ0257-6050 & 44.3354 & -60.8450 & 4.76 & $\ldots$ & $0.48 \pm 0.03$ & 1 & $\ldots$ & $\ldots$ & $\ldots$ & 44.3386 & -60.8358 & 2 \\
\hline SPT-CLJ0258-5756 & 44.5563 & -57.9438 & 4.50 & $\ldots$ & $>1.0$ & $\ldots$ & $\ldots$ & $\ldots$ & 17.4 & $\ldots$ & $\ldots$ & 1,2 \\
\hline SPT-CLJ0300-6315 & 45.1430 & -63.2643 & 4.88 & $\ldots$ & $>1.5$ & $\ldots$ & $\ldots$ & 31.6 & 63.6 & $\ldots$ & $\ldots$ & 1 \\
\hline SPT-CLJ0301-6456 & 45.4780 & -64.9470 & 4.94 & $\ldots$ & $0.65 \pm 0.03$ & 1 & $\ldots$ & $\ldots$ & $\ldots$ & 45.4809 & -64.9492 & 1 \\
\hline SPT-CLJ0307-6226 & 46.8335 & -62.4336 & 8.32 & $\ldots$ & $0.61 \pm 0.03$ & 1 & $\ldots$ & $\ldots$ & $\ldots$ & 46.8495 & -62.4028 & 3 \\
\hline SPT-CLJ0311-6354 & 47.8283 & -63.9083 & 7.33 & $\ldots$ & $0.30 \pm 0.02$ & 1 & $\ldots$ & $\ldots$ & $\ldots$ & 47.8229 & -63.9157 & 1 \\
\hline SPT-CLJ0313-5645 & 48.2604 & -56.7554 & 4.82 & $\ldots$ & $0.63 \pm 0.03$ & 1 & $\ldots$ & $\ldots$ & $\ldots$ & 48.2912 & -56.7420 & 1 \\
\hline SPT-CLJ0316-6059 & 49.2179 & -60.9849 & 4.59 & $\ldots$ & $>1.5$ & $\ldots$ & $\ldots$ & 26.4 & 26.9 & $\ldots$ & $\ldots$ & 1 \\
\hline SPT-CLJ0317-5935 & 49.3208 & -59.5856 & 5.91 & 0.469 & $0.47 \pm 0.02$ & 1 & $\ldots$ & $\ldots$ & $\ldots$ & 49.3160 & -59.5915 & 1 \\
\hline SPT-CLJ0320-5800 & 50.0316 & -58.0084 & 4.54 & $\ldots$ & $>1.0$ & $\ldots$ & $\ldots$ & $\ldots$ & 38.1 & $\ldots$ & $\ldots$ & 1,2 \\
\hline SPT-CLJ0324-6236 & 51.0530 & -62.6018 & 8.59 & $\ldots$ & $0.74 \pm 0.03$ & 1 & $\ldots$ & 0.3 & 0.0 & 51.0511 & -62.5988 & 3 \\
\hline SPT-CLJ0328-5541 & 52.1663 & -55.6975 & 7.08 & 0.084 & $0.10 \pm 0.03$ & 1 & $\ldots$ & $\ldots$ & $\ldots$ & 52.1496 & -55.7124 & 1 \\
\hline SPT-CLJ0333-5842 & 53.3195 & -58.7019 & 4.54 & $\ldots$ & $0.49 \pm 0.04$ & $3^{*}$ & $\ldots$ & $\ldots$ & $\ldots$ & 53.3322 & -58.7060 & 5 \\
\hline SPT-CLJ0337-6207 & 54.4720 & -62.1176 & 4.88 & $\ldots$ & $>1.3$ & $\ldots$ & 18.2 & $\ldots$ & 0.5 & . . & $\ldots$ & 1 \\
\hline SPT-CLJ0337-6300 & 54.4685 & -63.0098 & 5.29 & $\ldots$ & $0.46 \pm 0.03$ & 1 & $\ldots$ & $\ldots$ & $\ldots$ & 54.4744 & -63.0155 & 1 \\
\hline SPT-CLJ0341-5731 & 55.3979 & -57.5233 & 5.35 & $\ldots$ & $0.64 \pm 0.02$ & 1 & $\ldots$ & $\ldots$ & $\ldots$ & 55.3955 & -57.5244 & 1 \\
\hline
\end{tabular}


Table 3

Continued)

\begin{tabular}{|c|c|c|c|c|c|c|c|c|c|c|c|c|}
\hline \multirow[t]{2}{*}{ SPT ID } & \multicolumn{2}{|c|}{ Position } & \multirow[t]{2}{*}{$\xi$} & \multirow[t]{2}{*}{$z_{\text {spec }^{\mathrm{a}}}$} & \multirow{2}{*}{$\begin{array}{c}z_{\text {comb }} \pm \sigma_{z_{\text {comb }}} \\
\text { (or redshift lower limit) }\end{array}$} & \multirow[t]{2}{*}{ Flag $^{\mathrm{b}}$} & \multicolumn{3}{|c|}{ NIR Blank Field Probability $P_{\text {blank }}(\%)^{\mathrm{c}}$} & \multicolumn{2}{|c|}{ rBCG Position } & \multirow[t]{2}{*}{ Imaging Ref. ${ }^{\mathrm{d}}$} \\
\hline & R.A. (deg) & Decl. (deg) & & & & & NEWFIRM & Spitzer & WISE & R.A. (deg) & Decl. (deg) & \\
\hline SPT-CLJ0341-6143 & 55.3485 & -61.7192 & 5.60 & $\ldots$ & $0.63 \pm 0.03$ & 1 & $\ldots$ & $\ldots$ & $\ldots$ & 55.3488 & -61.7208 & 1 \\
\hline SPT-CLJ0343-5518 & 55.7634 & -55.3049 & 5.98 & $\ldots$ & $0.49 \pm 0.02$ & 1 & $\ldots$ & $\ldots$ & $\ldots$ & 55.7581 & -55.3111 & 1 \\
\hline SPT-CLJ0344-5452 & 56.0926 & -54.8726 & 5.41 & $\ldots$ & $1.01 \pm 0.07$ & 3 & $\ldots$ & 8.0 & 17.6 & $\ldots$ & $\ldots$ & 1 \\
\hline SPT-CLJ0344-5518 & 56.2101 & -55.3036 & 5.02 & $\ldots$ & $0.36 \pm 0.02$ & 1 & $\ldots$ & $\ldots$ & $\ldots$ & 56.1816 & -55.3179 & 1 \\
\hline SPT-CLJ0345-6419 & 56.2518 & -64.3326 & 5.57 & $\ldots$ & $0.93 \pm 0.07$ & 3 & 3.4 & 0.3 & 0.1 & 56.2518 & -64.3343 & 1 \\
\hline SPT-CLJ0346-5839 & 56.5746 & -58.6535 & 4.96 & $\ldots$ & $0.74 \pm 0.07$ & 3 & $\ldots$ & 0.4 & 0.4 & 56.5754 & -58.6532 & 1 \\
\hline SPT-CLJ0351-5636 & 57.9312 & -56.6099 & 4.65 & $\ldots$ & $0.38 \pm 0.03$ & 1 & $\ldots$ & $\ldots$ & $\ldots$ & 57.9446 & -56.6349 & 1 \\
\hline SPT-CLJ0351-5944 & 57.8654 & -59.7457 & 4.61 & $\ldots$ & $>1.0$ & ... & 20.4 & $\ldots$ & 60.4 & $\ldots$ & $\ldots$ & 2 \\
\hline SPT-CLJ0352-5647 & 58.2366 & -56.7992 & 7.11 & $\ldots$ & $0.66 \pm 0.03$ & 1 & $\ldots$ & $\ldots$ & $\ldots$ & 58.2759 & -56.7608 & 3 \\
\hline SPT-CLJ0354-5904 & 58.5611 & -59.0741 & 6.49 & $\ldots$ & $0.41 \pm 0.03$ & 1 & $\ldots$ & $\ldots$ & $\ldots$ & 58.6166 & -59.0971 & 1 \\
\hline SPT-CLJ0354-6032 ${ }^{\mathrm{f}}$ & 58.6744 & -60.5386 & 4.57 & $\ldots$ & $1.06 \pm 0.07$ & 3 & $\ldots$ & 8.1 & 1.9 & 58.6604 & -60.5462 & 2 \\
\hline SPT-CLJ0402-6129 & 60.7066 & -61.4988 & 4.83 & $\ldots$ & $0.53 \pm 0.04$ & 3 & $\ldots$ & $\ldots$ & $\ldots$ & 60.7213 & -61.4973 & 5 \\
\hline SPT-CLJ0403-5534 & 60.9479 & -55.5829 & 4.88 & $\ldots$ & $>1.5$ & $\ldots$ & $\ldots$ & 71.1 & 60.5 & $\ldots$ & $\ldots$ & 1 \\
\hline SPT-CLJ0403-5719 & 60.9670 & -57.3241 & 5.75 & $\ldots$ & $0.43 \pm 0.02$ & 1 & $\ldots$ & $\ldots$ & $\ldots$ & 60.9679 & -57.3285 & 1 \\
\hline SPT-CLJ0404-6510 & 61.0556 & -65.1817 & 4.75 & $\ldots$ & $0.15 \pm 0.02$ & 1 & $\ldots$ & $\ldots$ & $\ldots$ & 61.0934 & -65.1703 & 1 \\
\hline SPT-CLJ0406-5455 & 61.6922 & -54.9205 & 5.82 & $\ldots$ & $0.73 \pm 0.03$ & 1 & $\ldots$ & 3.3 & 19.6 & 61.6857 & -54.9257 & 1 \\
\hline SPT-CLJ0410-5454 & 62.6154 & -54.9016 & 5.06 & $\ldots$ & $>1.0$ & $\ldots$ & 76.8 & $\ldots$ & 35.0 & $\cdots$ & $\ldots$ & 1 \\
\hline SPT-CLJ0410-6343 & 62.5158 & -63.7285 & 5.79 & $\ldots$ & $0.49 \pm 0.02$ & 1 & $\ldots$ & $\ldots$ & $\ldots$ & 62.5207 & -63.7311 & 1 \\
\hline SPT-CLJ0411-5751 & 62.8433 & -57.8636 & 5.16 & $\ldots$ & $0.75 \pm 0.02$ & 1 & $\ldots$ & $\ldots$ & 25.3 & 62.8174 & -57.8517 & 1 \\
\hline SPT-CLJ0411-6340 & 62.8597 & -63.6810 & 6.41 & $\ldots$ & $0.14 \pm 0.02$ & 1 & $\ldots$ & $\ldots$ & $\ldots$ & 62.8676 & -63.6853 & 1 \\
\hline SPT-CLJ0412-5743 & 63.0245 & -57.7202 & 5.29 & $\ldots$ & $0.38 \pm 0.03$ & 1 & $\ldots$ & $\ldots$ & $\ldots$ & 63.0442 & -57.7383 & 1 \\
\hline SPT-CLJ0416-6359 & 64.1618 & -63.9964 & 6.06 & $\ldots$ & $0.30 \pm 0.02$ & 1 & $\ldots$ & $\ldots$ & $\ldots$ & 64.1735 & -64.0060 & 1 \\
\hline SPT-CLJ0423-5506 & 65.8153 & -55.1036 & 4.51 & $\ldots$ & $0.21 \pm 0.04$ & 3 & $\ldots$ & $\ldots$ & $\ldots$ & 65.8108 & -55.1143 & 5 \\
\hline SPT-CLJ0423-6143 & 65.9366 & -61.7183 & 4.65 & $\ldots$ & $0.71 \pm 0.04$ & 3 & $\ldots$ & $\ldots$ & 13.9 & 65.9323 & -61.7293 & 5 \\
\hline SPT-CLJ0426-5455 & 66.5205 & -54.9201 & 8.86 & $\ldots$ & $0.63 \pm 0.03$ & 1 & $\ldots$ & $\ldots$ & $\ldots$ & 66.5171 & -54.9253 & 3 \\
\hline SPT-CLJ0428-6049 & 67.0291 & -60.8302 & 5.06 & $\ldots$ & $>1.1$ & $\ldots$ & 69.0 & $\ldots$ & 0.5 & $\ldots$ & $\ldots$ & 1 \\
\hline SPT-CLJ0430-6251 $\mathrm{g}$ & 67.7086 & -62.8536 & 5.20 & $\ldots$ & $0.38 \pm 0.04$ & 4 & $\ldots$ & $\ldots$ & $\ldots$ & $\ldots$ & $\ldots$ & 1 \\
\hline SPT-CLJ0431-6126 & 67.8393 & -61.4438 & 6.40 & 0.058 & $0.08 \pm 0.02$ & 1 & $\ldots$ & $\ldots$ & $\ldots$ & 67.8053 & -61.4533 & 1 \\
\hline SPT-CLJ0433-5630 & 68.2522 & -56.5038 & 5.35 & 0.692 & $0.65 \pm 0.03$ & 1 & $\ldots$ & $\ldots$ & $\ldots$ & 68.2545 & -56.5190 & 1 \\
\hline SPT-CLJ0441-5859 & 70.4411 & -58.9931 & 4.54 & $\ldots$ & $>1.1$ & $\ldots$ & $\ldots$ & $\ldots$ & 27.7 & $\ldots$ & $\ldots$ & 5 \\
\hline SPT-CLJ0444-5603 & 71.1130 & -56.0566 & 5.30 & $\ldots$ & $0.98 \pm 0.07$ & 3 & 0.4 & 0.0 & 0.0 & 71.1077 & -56.0556 & 1 \\
\hline SPT-CLJ0446-5849 & 71.5160 & -58.8226 & 7.44 & $\ldots$ & $1.16 \pm 0.07$ & 3 & $\ldots$ & 0.9 & 5.6 & 71.5138 & -58.8247 & 1 \\
\hline SPT-CLJ0452-5945 & 73.1282 & -59.7622 & 4.50 & $\ldots$ & $>0.7$ & $\ldots$ & $\ldots$ & $\ldots$ & 39.4 & $\ldots$ & $\ldots$ & 5 \\
\hline SPT-CLJ0456-5623 & 74.1745 & -56.3868 & 4.76 & $\ldots$ & $0.66 \pm 0.03$ & 1 & $\ldots$ & $\ldots$ & $\ldots$ & $\ldots$ & $\ldots$ & 2 \\
\hline SPT-CLJ0456-6141 & 74.1496 & -61.6840 & 4.84 & $\ldots$ & $0.41 \pm 0.03$ & $3^{*}$ & $\ldots$ & $\ldots$ & $\ldots$ & 74.1361 & -61.6902 & 5 \\
\hline SPT-CLJ0458-5741 & 74.6021 & -57.6952 & 4.91 & $\ldots$ & $>1.0$ & $\ldots$ & $\ldots$ & $\ldots$ & 52.5 & $\ldots$ & $\ldots$ & 2 \\
\hline SPT-CLJ0502-6113 & 75.5400 & -61.2315 & 5.09 & $\ldots$ & $0.66 \pm 0.03$ & 1 & $\ldots$ & $\ldots$ & $\ldots$ & 75.5630 & -61.2314 & 1 \\
\hline SPT-CLJ0509-5342 & 77.3360 & -53.7046 & 6.61 & 0.461 & $0.43 \pm 0.02$ & 1 & $\ldots$ & $\ldots$ & $\ldots$ & 77.3392 & -53.7036 & 1,2 \\
\hline SPT-CLJ0511-5154 & 77.9202 & -51.9044 & 5.63 & 0.645 & $0.63 \pm 0.03$ & 1 & $\ldots$ & $\ldots$ & $\ldots$ & $\ldots$ & $\ldots$ & $1^{*}$ \\
\hline SPT-CLJ0514-5118 & 78.6859 & -51.3100 & 4.82 & $\ldots$ & $>1.2$ & ... & 28.2 & $\ldots$ & 49.7 & $\ldots$ & $\ldots$ & 1 \\
\hline SPT-CLJ0516-5430 & 79.1480 & -54.5062 & 9.42 & 0.295 & $0.31 \pm 0.02$ & 1 & $\ldots$ & $\ldots$ & $\ldots$ & 79.1557 & -54.5007 & 1,2 \\
\hline SPT-CLJ0521-5104 & 80.2983 & -51.0812 & 5.45 & 0.675 & $0.64 \pm 0.02$ & 1 & $\ldots$ & $\ldots$ & $\ldots$ & 80.3106 & -51.0718 & $1^{*}$ \\
\hline
\end{tabular}


Table 3

Continued)

\begin{tabular}{|c|c|c|c|c|c|c|c|c|c|c|c|c|}
\hline \multirow[t]{2}{*}{ SPT ID } & \multicolumn{2}{|c|}{ Position } & \multirow[t]{2}{*}{$\xi$} & \multirow[t]{2}{*}{$z_{\text {spec }^{\mathrm{a}}}$} & \multirow{2}{*}{$\begin{array}{c}z_{\text {comb }} \pm \sigma_{z_{\text {comb }}} \\
\text { (or redshift lower limit) }\end{array}$} & \multirow[t]{2}{*}{ Flag $^{\mathrm{b}}$} & \multicolumn{3}{|c|}{ NIR Blank Field Probability $P_{\text {blank }}(\%)^{\mathrm{c}}$} & \multicolumn{2}{|c|}{ rBCG Position } & \multirow[t]{2}{*}{ Imaging Ref. ${ }^{\mathrm{C}}$} \\
\hline & R.A. (deg) & Decl. (deg) & & & & & NEWFIRM & Spitzer & WISE & R.A. (deg) & Decl. (deg) & \\
\hline SPT-CLJ0522-5026 & 80.5190 & -50.4409 & 4.87 & $\ldots$ & $0.51 \pm 0.03$ & 1 & $\cdots$ & $\cdots$ & & 80.5000 & -50.4696 & $11^{*}$ \\
\hline SPT-CLJ0527-5928 & 81.8111 & -59.4833 & 4.71 & $\ldots$ & $>0.9$ & $\ldots$ & $\ldots$ & $\ldots$ & 25.5 & & & 2 \\
\hline SPT-CLJ0528-5300 & 82.0173 & -53.0001 & 5.45 & 0.768 & $0.77 \pm 0.03$ & 1 & $\ldots$ & 0.0 & 0.0 & 82.0221 & -52.9982 & $1^{*}$ \\
\hline SPT-CLJ0529-5238 & 82.2923 & -52.6417 & 4.52 & $\ldots$ & $>1.1$ & $\ldots$ & $\ldots$ & $\ldots$ & 81.8 & $\ldots$ & $\ldots$ & $1^{*}$ \\
\hline SPT-CLJ0532-5647 & 83.1586 & -56.7893 & 4.51 & $\ldots$ & $>0.9$ & $\ldots$ & $\ldots$ & $\ldots$ & 39.0 & $\ldots$ & $\ldots$ & 2 \\
\hline SPT-CLJ0533-5005 & 83.3984 & -50.0918 & 5.59 & 0.881 & $0.81 \pm 0.03$ & 1 & $\ldots$ & 0.0 & 18.4 & 83.4144 & -50.0845 & 2 \\
\hline SPT-CLJ0534-5937 & 83.6018 & -59.6289 & 4.57 & 0.576 & $0.57 \pm 0.02$ & 1 & $\ldots$ & $\ldots$ & & 83.6255 & -59.6152 & 2 \\
\hline SPT-CLJ0537-5549 & 84.2578 & -55.8268 & 4.55 & $\ldots$ & $>1.1$ & $\ldots$ & $\ldots$ & $\ldots$ & 28.4 & $\ldots$ & $\ldots$ & 2 \\
\hline SPT-CLJ0538-5657 & 84.5865 & -56.9530 & 4.63 & $\ldots$ & $>1.5$ & $\ldots$ & $\ldots$ & 21.8 & 25.1 & $\ldots$ & $\ldots$ & 2 \\
\hline SPT-CLJ0539-5744 & 84.9998 & -57.7432 & 5.12 & $\ldots$ & $0.76 \pm 0.03$ & 1 & $\ldots$ & 0.0 & 0.0 & 84.9950 & -57.7424 & 2 \\
\hline SPT-CLJ0546-5345 & 86.6541 & -53.7615 & 7.69 & 1.066 & $1.04 \pm 0.07$ & 3 & $\ldots$ & 0.0 & 0.0 & 86.6569 & -53.7587 & $1^{*}$ \\
\hline SPT-CLJ0551-5709 & 87.9016 & -57.1565 & 6.13 & 0.423 & $0.43 \pm 0.02$ & 1 & $\ldots$ & $\ldots$ & $\ldots$ & 87.8981 & -57.1414 & 2 \\
\hline SPT-CLJ0556-5403 & 89.2016 & -54.0630 & 4.83 & $\ldots$ & $0.93 \pm 0.04$ & 4 & 17.1 & $\ldots$ & 0.0 & 89.2018 & -54.0582 & 2 \\
\hline SPT-CLJ0559-5249 & 89.9245 & -52.8265 & 9.28 & 0.609 & $0.63 \pm 0.02$ & 1 & $\ldots$ & $\ldots$ & $\ldots$ & 89.9301 & -52.8242 & 2 \\
\hline SPT-CLJ2002-5335 & 300.5113 & -53.5913 & 4.53 & $\ldots$ & $>1.0$ & $\ldots$ & $\ldots$ & $\ldots$ & 7.5 & $\ldots$ & $\ldots$ & 1 \\
\hline SPT-CLJ2005-5635 & 301.3385 & -56.5902 & 4.68 & $\ldots$ & $>0.6$ & $\ldots$ & $\ldots$ & $\ldots$ & 71.3 & $\ldots$ & $\ldots$ & 1 \\
\hline SPT-CLJ2006-5325 & 301.6620 & -53.4286 & 5.06 & $\ldots$ & $>1.5$ & $\ldots$ & 66.8 & 52.3 & 71.8 & $\ldots$ & $\ldots$ & 1 \\
\hline SPT-CLJ2007-4906 & 301.9663 & -49.1105 & 4.50 & $\ldots$ & $1.25 \pm 0.07$ & 3 & $\ldots$ & 0.9 & 5.9 & 301.9692 & -49.1085 & 1 \\
\hline SPT-CLJ2009-5756 & 302.4261 & -57.9480 & 4.68 & $\ldots$ & $0.63 \pm 0.03$ & 1 & $\ldots$ & $\ldots$ & $\ldots$ & $\ldots$ & $\ldots$ & 1 \\
\hline SPT-CLJ2011-5228 & 302.7810 & -52.4734 & 4.55 & $\ldots$ & $0.96 \pm 0.04$ & 1 & $\ldots$ & $\ldots$ & 51.6 & 302.7814 & -52.4709 & 1 \\
\hline SPT-CLJ2011-5725 & 302.8526 & -57.4214 & 5.43 & 0.279 & $0.28 \pm 0.03$ & 1 & $\ldots$ & $\ldots$ & $\ldots$ & 302.8624 & -57.4197 & 1 \\
\hline SPT-CLJ2012-5342 & 303.0822 & -53.7137 & 4.65 & $\ldots$ & $>0.7$ & $\ldots$ & $\ldots$ & $\ldots$ & 11.0 & $\ldots$ & $\ldots$ & 1 \\
\hline SPT-CLJ2012-5649 & 303.1132 & -56.8308 & 5.99 & 0.055 & $0.07 \pm 0.02$ & 1 & $\ldots$ & $\ldots$ & $\ldots$ & 303.1142 & -56.8270 & 2 \\
\hline SPT-CLJ2013-5432 & 303.4968 & -54.5445 & 4.75 & $\ldots$ & $>1.0$ & $\ldots$ & 49.7 & $\ldots$ & 63.4 & $\ldots$ & $\ldots$ & 1 \\
\hline SPT-CLJ2015-5504 & 303.9884 & -55.0715 & 4.64 & $\ldots$ & $>0.6$ & $\ldots$ & 87.3 & $\ldots$ & 67.0 & $\ldots$ & $\ldots$ & 1 \\
\hline SPT-CLJ2016-4954 & 304.0181 & -49.9122 & 5.01 & $\ldots$ & $0.26 \pm 0.02$ & 1 & $\ldots$ & $\ldots$ & $\ldots$ & 304.0067 & -49.9067 & 1 \\
\hline SPT-CLJ2017-6258 & 304.4827 & -62.9763 & 6.45 & $\ldots$ & $0.57 \pm 0.03$ & 1 & $\ldots$ & $\ldots$ & $\ldots$ & 304.4730 & -62.9950 & 3 \\
\hline SPT-CLJ2018-4528 & 304.6076 & -45.4807 & 4.64 & $\ldots$ & $0.40 \pm 0.03$ & 1 & $\ldots$ & $\ldots$ & $\ldots$ & 304.6164 & -45.4761 & 1 \\
\hline SPT-CLJ2019-5642 & 304.7703 & -56.7079 & 5.25 & $\ldots$ & $0.15 \pm 0.03$ & 1 & $\ldots$ & $\ldots$ & $\ldots$ & 304.8137 & -56.7122 & 2 \\
\hline SPT-CLJ2020-4646 & 305.1936 & -46.7702 & 5.09 & $\ldots$ & $0.17 \pm 0.02$ & 1 & $\ldots$ & $\ldots$ & $\ldots$ & 305.1973 & -46.7748 & 1 \\
\hline SPT-CLJ2020-6314 & 305.0301 & -63.2413 & 5.37 & $\ldots$ & $0.58 \pm 0.02$ & 1 & $\ldots$ & $\ldots$ & $\ldots$ & 305.0350 & -63.2471 & 2 \\
\hline SPT-CLJ2021-5256 & 305.4690 & -52.9439 & 5.31 & & $0.11 \pm 0.02$ & 1 & $\ldots$ & $\ldots$ & $\ldots$ & 305.4725 & -52.9509 & 1 \\
\hline SPT-CLJ2022-6323 & 305.5235 & -63.3973 & 6.58 & 0.383 & $0.41 \pm 0.02$ & 1 & $\ldots$ & $\ldots$ & $\ldots$ & 305.5410 & -63.3971 & 2,4 \\
\hline SPT-CLJ2023-5535 & 305.8377 & -55.5903 & 13.41 & 0.232 & $0.22 \pm 0.02$ & 1 & $\ldots$ & $\ldots$ & $\ldots$ & 305.9069 & -55.5697 & 2,3 \\
\hline SPT-CLJ2025-5117 & 306.4836 & -51.2904 & 9.48 & $\ldots$ & $0.20 \pm 0.02$ & 1 & $\ldots$ & $\ldots$ & $\ldots$ & 306.4822 & -51.2744 & 1 \\
\hline SPT-CLJ2026-4513 & 306.6140 & -45.2256 & 5.53 & $\ldots$ & $0.71 \pm 0.03$ & 1 & $\ldots$ & 2.1 & 18.8 & 306.6180 & -45.2338 & 1 \\
\hline SPT-CLJ2030-5638 & 307.7067 & -56.6352 & 5.47 & $\ldots$ & $0.39 \pm 0.03$ & 1 & $\cdots$ & $\ldots$ & $\ldots$ & 307.6886 & -56.6322 & 2,4 \\
\hline SPT-CLJ2032-5627 & 308.0800 & -56.4557 & 8.14 & 0.284 & $0.33 \pm 0.02$ & 1 & $\cdots$ & $\cdots$ & $\ldots$ & 308.0586 & -56.4368 & 2 \\
\hline SPT-CLJ2034-5936 & 308.5408 & -59.6007 & 8.57 & $\ldots$ & $0.92 \pm 0.07$ & 3 & $\cdots$ & 0.2 & 25.9 & 308.5414 & -59.6034 & 2 \\
\hline SPT-CLJ2035-5251 & 308.8026 & -52.8527 & 9.99 & $\cdots$ & $0.47 \pm 0.02$ & 1 & $\cdots$ & $\ldots$ & $\cdots$ & $\cdots$ & $\ldots$ & 1 \\
\hline SPT-CLJ2035-5614 & 308.9023 & -56.2407 & 4.55 & $\ldots$ & $>1.0$ & $\ldots$ & $\ldots$ & $\ldots$ & 0.1 & $\ldots$ & $\ldots$ & 1 \\
\hline SPT-CLJ2039-5723 & 309.8246 & -57.3871 & 4.69 & $\ldots$ & $>1.2$ & $\ldots$ & 9.2 & $\ldots$ & 1.2 & $\ldots$ & $\ldots$ & 1,2 \\
\hline
\end{tabular}


Table 3

Continued)

\begin{tabular}{|c|c|c|c|c|c|c|c|c|c|c|c|c|}
\hline \multirow[t]{2}{*}{ SPT ID } & \multicolumn{2}{|c|}{ Position } & \multirow[t]{2}{*}{$\xi$} & \multirow[t]{2}{*}{$z_{\text {spec }^{\mathrm{a}}}$} & \multirow{2}{*}{$\begin{array}{c}z_{\text {comb }} \pm \sigma_{z_{\text {comb }}} \\
\text { (or redshift lower limit) }\end{array}$} & \multirow[t]{2}{*}{ Flag $^{\mathrm{b}}$} & \multicolumn{3}{|c|}{ NIR Blank Field Probability $P_{\text {blank }}(\%)^{\mathrm{c}}$} & \multicolumn{2}{|c|}{ rBCG Position } & \multirow[t]{2}{*}{ Imaging Ref. } \\
\hline & R.A. (deg) & Decl. (deg) & & & & & NEWFIRM & Spitzer & WISE & R.A. (deg) & Decl. (deg) & \\
\hline SPT-CLJ2040-4451 & 310.2468 & -44.8599 & 6.28 & $\ldots$ & $1.35 \pm 0.07$ & 3 & 29.9 & 4.6 & 3.6 & 310.2384 & -44.8593 & 1 \\
\hline SPT-CLJ2040-5230 & 310.1255 & -52.5052 & 4.70 & $\ldots$ & $>1.0$ & $\ldots$ & 44.4 & $\ldots$ & 20.2 & $\ldots$ & $\ldots$ & 1 \\
\hline SPT-CLJ2040-5342 & 310.2195 & -53.7122 & 5.88 & $\ldots$ & $0.57 \pm 0.04$ & 1 & $\ldots$ & $\ldots$ & $\ldots$ & $\ldots$ & $\ldots$ & 1 \\
\hline SPT-CLJ2040-5725 & 310.0631 & -57.4287 & 6.38 & 0.930 & $0.91 \pm 0.07$ & 3 & $\ldots$ & 0.9 & 15.0 & 310.0552 & -57.4209 & 1,2 \\
\hline SPT-CLJ2043-5035 & 310.8285 & -50.5929 & 7.81 & 0.723 & $0.77 \pm 0.03$ & 1 & $\ldots$ & 0.6 & 0.4 & $\ldots$ & $\ldots$ & 1 \\
\hline SPT-CLJ2043-5614 & 310.7906 & -56.2351 & 4.72 & $\ldots$ & $0.69 \pm 0.03$ & 1 & $\ldots$ & $\ldots$ & $\ldots$ & 310.7788 & -56.2390 & 1 \\
\hline SPT-CLJ2045-6026 & 311.3649 & -60.4469 & 4.77 & $\ldots$ & $>0.5$ & $\ldots$ & 86.3 & $\ldots$ & 94.6 & $\ldots$ & $\ldots$ & 1 \\
\hline SPT-CLJ2046-4542 & 311.5620 & -45.7111 & 4.54 & $\ldots$ & $>1.0$ & $\ldots$ & $\ldots$ & $\ldots$ & 65.3 & $\ldots$ & $\ldots$ & 1 \\
\hline SPT-CLJ2048-4524 & 312.2268 & -45.4150 & 4.56 & $\ldots$ & $>1.0$ & $\ldots$ & $\ldots$ & $\ldots$ & 96.6 & $\ldots$ & $\ldots$ & 1 \\
\hline SPT-CLJ2051-6256 & 312.8027 & -62.9348 & 5.17 & $\ldots$ & $0.47 \pm 0.02$ & 1 & $\ldots$ & $\ldots$ & $\ldots$ & 312.8230 & -62.9407 & 1 \\
\hline SPT-CLJ2055-5456 & 313.9941 & -54.9366 & 6.61 & $\ldots$ & $0.11 \pm 0.02$ & 1 & $\ldots$ & $\ldots$ & $\ldots$ & 313.9838 & -54.9273 & 2 \\
\hline SPT-CLJ2056-5106 & 314.0723 & -51.1163 & 4.70 & $\ldots$ & $>1.0$ & $\ldots$ & 43.0 & $\ldots$ & 45.4 & $\ldots$ & $\ldots$ & 1 \\
\hline SPT-CLJ2056-5459 & 314.2199 & -54.9892 & 6.05 & 0.718 & $0.84 \pm 0.07$ & 3 & $\ldots$ & 0.3 & 9.3 & 314.2232 & -54.9858 & 2 \\
\hline SPT-CLJ2057-5251 & 314.4105 & -52.8567 & 4.52 & $\ldots$ & $>1.5$ & $\ldots$ & $\ldots$ & 82.1 & 65.8 & $\ldots$ & $\ldots$ & 1 \\
\hline SPT-CLJ2058-5608 & 314.5893 & -56.1454 & 5.02 & 0.606 & $0.59 \pm 0.02$ & 1 & $\ldots$ & $\ldots$ & $\ldots$ & 314.5930 & -56.1464 & 1 \\
\hline SPT-CLJ2059-5018 & 314.9324 & -50.3049 & 4.79 & $\ldots$ & $0.39 \pm 0.02$ & 1 & $\ldots$ & $\ldots$ & $\ldots$ & 314.9220 & -50.3029 & 1 \\
\hline SPT-CLJ2100-4548 & 315.0936 & -45.8057 & 4.84 & 0.712 & $0.74 \pm 0.03$ & 1 & $\ldots$ & 1.2 & 6.9 & $\ldots$ & $\ldots$ & 1 \\
\hline SPT-CLJ2100-5708 & 315.1502 & -57.1347 & 5.11 & $\ldots$ & $0.59 \pm 0.03$ & 1 & $\ldots$ & $\ldots$ & $\ldots$ & 315.1470 & -57.1385 & 1 \\
\hline SPT-CLJ2101-5542 & 315.3106 & -55.7027 & 5.04 & $\ldots$ & $0.22 \pm 0.02$ & 1 & $\ldots$ & $\ldots$ & $\ldots$ & 315.3040 & -55.6940 & 1 \\
\hline SPT-CLJ2101-6123 & 315.4594 & -61.3972 & 5.28 & $\ldots$ & $0.60 \pm 0.03$ & 1 & $\ldots$ & $\ldots$ & $\ldots$ & 315.4326 & -61.4047 & 1 \\
\hline SPT-CLJ2103-5411 & 315.7687 & -54.1951 & 4.88 & $\ldots$ & $0.46 \pm 0.02$ & 1 & $\ldots$ & $\ldots$ & $\ldots$ & 315.7792 & -54.1945 & 1 \\
\hline SPT-CLJ2104-5224 & 316.2283 & -52.4044 & 5.32 & 0.799 & $0.81 \pm 0.03$ & 1 & $\ldots$ & 65.7 & 3.0 & 316.2120 & -52.4079 & 1 \\
\hline SPT-CLJ2106-5820 & 316.5144 & -58.3459 & 4.81 & $\ldots$ & $>1.0$ & $\ldots$ & 79.4 & $\ldots$ & 60.6 & $\ldots$ & $\ldots$ & 1 \\
\hline SPT-CLJ2106-5844 & 316.5210 & -58.7448 & 22.08 & 1.132 & $1.20 \pm 0.07$ & 3 & $\ldots$ & 0.0 & 0.1 & 316.5194 & -58.7412 & 2 \\
\hline SPT-CLJ2106-6019 & 316.6642 & -60.3299 & 4.98 & $\ldots$ & $0.97 \pm 0.03$ & 1 & 2.5 & $\ldots$ & 1.0 & 316.6449 & -60.3385 & 1 \\
\hline SPT-CLJ2106-6303 & 316.6596 & -63.0510 & 4.90 & $\ldots$ & $>1.0$ & $\ldots$ & 19.6 & $\ldots$ & 16.7 & $\ldots$ & $\ldots$ & 1 \\
\hline SPT-CLJ2109-4626 & 317.4516 & -46.4370 & 5.51 & $\ldots$ & $0.98 \pm 0.09$ & 3 & $\ldots$ & 1.1 & 0.6 & 317.4557 & -46.4376 & 1 \\
\hline SPT-CLJ2109-5040 & 317.3820 & -50.6773 & 5.17 & $\ldots$ & $0.47 \pm 0.03$ & 1 & $\ldots$ & $\ldots$ & $\ldots$ & 317.4016 & -50.6815 & 1 \\
\hline SPT-CLJ2110-5244 & 317.5502 & -52.7486 & 6.22 & $\ldots$ & $0.61 \pm 0.02$ & 1 & $\ldots$ & $\ldots$ & $\ldots$ & 317.5520 & -52.7496 & 1 \\
\hline SPT-CLJ2111-5338 & 317.9217 & -53.6496 & 5.65 & $\ldots$ & $0.43 \pm 0.03$ & 1 & $\ldots$ & $\ldots$ & $\ldots$ & 317.9357 & -53.6477 & 1 \\
\hline SPT-CLJ2115-4659 & 318.7995 & -46.9862 & 5.60 & $\ldots$ & $0.34 \pm 0.02$ & 1 & $\ldots$ & $\ldots$ & $\ldots$ & 318.8064 & -46.9797 & 1 \\
\hline SPT-CLJ2118-5055 & 319.7291 & -50.9329 & 5.62 & 0.625 & $0.63 \pm 0.03$ & 1 & $\ldots$ & $\ldots$ & $\ldots$ & $\ldots$ & $\ldots$ & 1 \\
\hline SPT-CLJ2119-6230 & 319.8846 & -62.5096 & 4.55 & $\ldots$ & $0.72 \pm 0.03$ & 1 & $\ldots$ & $\ldots$ & $\ldots$ & 319.8765 & -62.5106 & 1 \\
\hline SPT-CLJ2120-4728k & 320.1594 & -47.4776 & 5.98 & $\ldots$ & $0.99 \pm 0.07$ & 3 & 8.7 & 0.5 & 2.1 & 320.1638 & -47.4750 & 1 \\
\hline SPT-CLJ2121-5546 & 320.2715 & -55.7780 & 4.79 & $\ldots$ & $>0.8$ & $\ldots$ & 11.5 & $\ldots$ & 0.9 & $\ldots$ & $\ldots$ & 2 \\
\hline SPT-CLJ2121-6335 & 320.4269 & -63.5843 & 5.43 & $\ldots$ & $0.23 \pm 0.02$ & 1 & $\ldots$ & $\ldots$ & $\ldots$ & 320.4303 & -63.5973 & 1 \\
\hline SPT-CLJ2124-6124 & 321.1488 & -61.4141 & 8.21 & 0.435 & $0.44 \pm 0.02$ & 1 & $\ldots$ & $\ldots$ & $\ldots$ & 321.1577 & -61.4077 & 1 \\
\hline SPT-CLJ2125-6113 & 321.2902 & -61.2292 & 4.74 & $\ldots$ & $>1.5$ & $\ldots$ & $\ldots$ & 91.8 & 17.2 & $\ldots$ & $\ldots$ & 1,2 \\
\hline SPT-CLJ2127-6443 & 321.9939 & -64.7288 & 4.54 & $\ldots$ & $>1.0$ & $\ldots$ & $\ldots$ & $\ldots$ & 80.2 & $\ldots$ & $\ldots$ & 1 \\
\hline SPT-CLJ2130-4737 & 322.6622 & -47.6257 & 4.83 & $\ldots$ & $>1.5$ & $\ldots$ & 76.9 & 22.4 & 68.5 & .. & $\ldots$ & 1 \\
\hline SPT-CLJ2130-6458 & 322.7285 & -64.9764 & 7.57 & 0.316 & $0.36 \pm 0.02$ & 1 & $\ldots$ & $\ldots$ & $\ldots$ & 322.7343 & -64.9779 & 1,2 \\
\hline SPT-CLJ2131-5003 & 322.9717 & -50.0647 & 4.83 & $\ldots$ & $0.45 \pm 0.02$ & 1 & $\ldots$ & $\ldots$ & $\ldots$ & 322.9637 & -50.0624 & 1 \\
\hline
\end{tabular}




\begin{tabular}{|c|c|c|c|c|c|c|c|c|c|c|c|c|}
\hline \multirow[t]{2}{*}{ SPT ID } & \multicolumn{2}{|c|}{ Position } & \multirow[t]{2}{*}{$\xi$} & \multirow[t]{2}{*}{$z_{\text {spec }}{ }^{\mathrm{a}}$} & \multirow{2}{*}{$\begin{array}{c}z_{\text {comb }} \pm \sigma_{z_{\text {comb }}} \\
\text { (or redshift lower limit) }\end{array}$} & \multirow[t]{2}{*}{ Flag $^{\mathrm{b}}$} & \multicolumn{3}{|c|}{ NIR Blank Field Probability $P_{\text {blank }}(\%)^{\mathrm{c}}$} & \multicolumn{2}{|c|}{ rBCG Position } & \multirow[t]{2}{*}{ Imaging Ref. $^{\mathrm{C}}$} \\
\hline & R.A. (deg) & Decl. (deg) & & & & & NEWFIRM & Spitzer & WISE & R.A. (deg) & Decl. (deg) & \\
\hline SPT-CLJ2133-5411 & 323.2978 & -54.1845 & 4.58 & $\cdots$ & $>1.5$ & $\ldots$ & $\ldots$ & 48.7 & 32.0 & $\ldots$ & $\ldots$ & 1 \\
\hline SPT-CLJ2135-5452 & 323.9060 & -54.8773 & 4.61 & & $>1.0$ & $\ldots$ & $\ldots$ & $\ldots$ & 53.4 & $\ldots$ & $\ldots$ & 1 \\
\hline SPT-CLJ2135-5726 & 323.9158 & -57.4415 & 10.43 & 0.427 & $0.46 \pm 0.02$ & 1 & $\ldots$ & $\ldots$ & $\ldots$ & 323.9059 & -57.4418 & 2,4 \\
\hline SPT-CLJ2136-4704 & 324.1175 & -47.0803 & 6.17 & 0.425 & $0.43 \pm 0.03$ & 1 & $\ldots$ & $\ldots$ & $\ldots$ & 324.1640 & -47.0716 & 1 \\
\hline SPT-CLJ2136-5519 & 324.2392 & -55.3215 & 4.65 & $\ldots$ & $>1.5$ & $\ldots$ & $\ldots$ & 35.2 & 62.1 & $\ldots$ & $\ldots$ & 1 \\
\hline SPT-CLJ2136-5535 & 324.0898 & -55.5853 & 4.58 & $\ldots$ & $>1.2$ & $\ldots$ & $\ldots$ & $\ldots$ & 5.2 & $\ldots$ & $\ldots$ & 1 \\
\hline SPT-CLJ2136-5723 & 324.1209 & -57.3923 & 4.55 & $\ldots$ & $>1.0$ & $\ldots$ & $\ldots$ & $\ldots$ & 40.0 & $\ldots$ & $\ldots$ & 2,4 \\
\hline SPT-CLJ2136-6307 & 324.2334 & -63.1233 & 6.25 & 0.926 & $1.00 \pm 0.07$ & 3 & $\ldots$ & 0.2 & 1.4 & 324.2239 & -63.1143 & 1,2 \\
\hline SPT-CLJ2137-6437 & 324.4178 & -64.6235 & 4.60 & $\ldots$ & $0.91 \pm 0.07$ & 3 & $\ldots$ & 3.7 & 12.8 & 324.4337 & -64.6234 & 1 \\
\hline SPT-CLJ2138-6007 & 324.5060 & -60.1324 & 12.64 & 0.319 & $0.34 \pm 0.02$ & 1 & $\ldots$ & $\ldots$ & $\ldots$ & 324.5036 & -60.1317 & 2,4 \\
\hline SPT-CLJ2139-5420 & 324.9669 & -54.3396 & 4.81 & $\ldots$ & $0.24 \pm 0.02$ & 1 & $\ldots$ & $\ldots$ & $\ldots$ & 324.9713 & -54.3410 & 1 \\
\hline SPT-CLJ2140-5331 & 325.0304 & -53.5199 & 4.55 & $\ldots$ & $0.51 \pm 0.02$ & 1 & $\ldots$ & $\ldots$ & $\ldots$ & 325.0287 & -53.5037 & 1 \\
\hline SPT-CLJ2140-5727 & 325.1380 & -57.4564 & 5.08 & $\ldots$ & $0.40 \pm 0.03$ & 1 & $\ldots$ & $\ldots$ & $\ldots$ & $\ldots$ & $\ldots$ & 2 \\
\hline SPT-CLJ2142-4846 & 325.5693 & -48.7743 & 4.53 & $\ldots$ & $>0.8$ & $\ldots$ & $\ldots$ & $\ldots$ & 96.1 & $\ldots$ & $\ldots$ & 1 \\
\hline SPT-CLJ2145-5644 & 326.4694 & -56.7477 & 12.30 & 0.480 & $0.48 \pm 0.02$ & 1 & $\ldots$ & $\ldots$ & $\ldots$ & 326.5298 & -56.7422 & 2 \\
\hline SPT-CLJ2146-4633 & 326.6473 & -46.5505 & 9.59 & 0.933 & $0.95 \pm 0.07$ & 3 & $\ldots$ & 0.2 & 1.5 & $\ldots$ & $\ldots$ & 1 \\
\hline SPT-CLJ2146-4846 & 326.5346 & -48.7774 & 5.88 & 0.623 & $0.62 \pm 0.02$ & 2 & $\ldots$ & $\ldots$ & $\ldots$ & 326.5246 & -48.7813 & 1 \\
\hline SPT-CLJ2146-5736 & 326.6963 & -57.6138 & 5.94 & $\ldots$ & $0.61 \pm 0.02$ & 1 & $\ldots$ & $\ldots$ & $\ldots$ & 326.6954 & -57.6310 & 2 \\
\hline SPT-CLJ2148-4843 & 327.0971 & -48.7287 & 4.64 & $\ldots$ & $0.98 \pm 0.07$ & 3 & $\ldots$ & 0.6 & 1.1 & $\ldots$ & $\ldots$ & 1 \\
\hline SPT-CLJ2148-6116 & 327.1798 & -61.2791 & 7.27 & 0.571 & $0.52 \pm 0.02$ & 1 & $\ldots$ & $\ldots$ & $\ldots$ & 327.1617 & -61.2655 & 1,2 \\
\hline SPT-CLJ2149-5330 & 327.3770 & -53.5014 & 4.79 & $\ldots$ & $0.60 \pm 0.03$ & 1 & $\ldots$ & $\ldots$ & $\ldots$ & 327.4331 & -53.5176 & 1 \\
\hline SPT-CLJ2150-6111 & 327.7177 & -61.1954 & 4.70 & $\ldots$ & $>1.1$ & $\ldots$ & 25.4 & $\ldots$ & 15.7 & $\ldots$ & $\ldots$ & 1 \\
\hline SPT-CLJ2152-4629 & 328.1943 & -46.4947 & 5.60 & $\ldots$ & $>1.5$ & $\ldots$ & 20.0 & 10.6 & 8.0 & $\ldots$ & $\ldots$ & 1 \\
\hline SPT-CLJ2152-5143 & 328.0034 & -51.7245 & 4.53 & $\ldots$ & $0.41 \pm 0.03$ & 1 & $\ldots$ & $\ldots$ & $\ldots$ & 327.9829 & -51.7226 & 1 \\
\hline SPT-CLJ2152-5633 & 328.1458 & -56.5641 & 5.84 & $\ldots$ & $>1.5$ & $\ldots$ & $\ldots$ & 20.2 & 55.5 & $\ldots$ & $\ldots$ & 1,2 \\
\hline SPT-CLJ2155-5103 & 328.8747 & -51.0508 & 4.52 & $\ldots$ & $>1.1$ & $\ldots$ & $\ldots$ & $\ldots$ & 34.1 & $\ldots$ & $\ldots$ & 1 \\
\hline SPT-CLJ2155-5225 & 328.8941 & -52.4169 & 4.77 & $\ldots$ & $0.62 \pm 0.03$ & 1 & $\ldots$ & $\ldots$ & $\ldots$ & 328.8997 & -52.4194 & 1 \\
\hline SPT-CLJ2155-6048 & 328.9850 & -60.8072 & 5.24 & 0.539 & $0.48 \pm 0.02$ & 1 & $\ldots$ & $\ldots$ & $\ldots$ & 328.9811 & -60.8174 & 1 \\
\hline SPT-CLJ2158-4702 & 329.6901 & -47.0348 & 4.56 & $\ldots$ & $>0.9$ & $\ldots$ & $\ldots$ & $\ldots$ & 64.4 & $\ldots$ & $\ldots$ & 1 \\
\hline SPT-CLJ2158-4851 & 329.5737 & -48.8536 & 4.61 & $\ldots$ & $>0.8$ & $\ldots$ & 36.3 & $\ldots$ & 58.5 & $\ldots$ & $\ldots$ & 1 \\
\hline SPT-CLJ2158-5615 & 329.5975 & -56.2588 & 4.54 & $\ldots$ & $>1.1$ & $\ldots$ & $\ldots$ & $\ldots$ & 53.6 & $\ldots$ & $\ldots$ & 1 \\
\hline SPT-CLJ2158-6319 & 329.6390 & -63.3175 & 4.54 & $\ldots$ & $>1.1$ & $\ldots$ & $\ldots$ & $\ldots$ & 99.7 & $\ldots$ & $\ldots$ & 1 \\
\hline SPT-CLJ2159-6244 & 329.9922 & -62.7420 & 6.08 & $\ldots$ & $0.43 \pm 0.02$ & 1 & $\ldots$ & $\ldots$ & $\ldots$ & 329.9944 & -62.7539 & 2 \\
\hline SPT-CLJ2200-5547 & 330.0304 & -55.7954 & 4.80 & $\ldots$ & $>1.0$ & $\ldots$ & 23.5 & $\ldots$ & 19.7 & $\ldots$ & $\ldots$ & 1 \\
\hline SPT-CLJ2201-5956 & 330.4727 & -59.9473 & 13.99 & 0.097 & $0.07 \pm 0.02$ & 1 & $\ldots$ & $\ldots$ & $\ldots$ & 330.4723 & -59.9454 & 2 \\
\hline SPT-CLJ2202-5936 & 330.5483 & -59.6021 & 4.89 & $\ldots$ & $0.42 \pm 0.03$ & 1 & $\ldots$ & $\ldots$ & $\ldots$ & 330.5522 & -59.6037 & 1 \\
\hline SPT-CLJ2259-5432 & 344.9820 & -54.5356 & 4.78 & $\ldots$ & $0.46 \pm 0.03$ & 1 & $\ldots$ & $\ldots$ & $\ldots$ & 344.9765 & -54.5260 & 3 \\
\hline SPT-CLJ2259-5617 & 344.9974 & -56.2877 & 5.29 & $\ldots$ & $0.15 \pm 0.02$ & 1 & $\ldots$ & $\ldots$ & $\ldots$ & 345.0044 & -56.2848 & 1,2 \\
\hline SPT-CLJ2300-5331 & 345.1765 & -53.5170 & 5.29 & 0.262 & $0.26 \pm 0.02$ & 1 & $\ldots$ & $\ldots$ & $\ldots$ & 345.1655 & -53.5199 & 2 \\
\hline SPT-CLJ2301-5046 & 345.4585 & -50.7823 & 4.58 & $\ldots$ & $>1.5$ & $\ldots$ & $\ldots$ & 64.3 & 83.7 & $\ldots$ & $\ldots$ & 1 \\
\hline SPT-CLJ2301-5546 & 345.4688 & -55.7758 & 5.19 & 0.748 & $0.74 \pm 0.03$ & 1 & $\cdots$ & 0.2 & 0.0 & 345.4595 & -55.7842 & 1 \\
\hline
\end{tabular}


Table 3

\begin{tabular}{|c|c|c|c|c|c|c|c|c|c|c|c|c|}
\hline \multirow[t]{2}{*}{ SPT ID } & \multicolumn{2}{|c|}{ Position } & \multirow[t]{2}{*}{$\xi$} & \multirow[t]{2}{*}{$z_{\text {spec }}{ }^{\mathrm{a}}$} & \multirow{2}{*}{$\begin{array}{c}z_{\text {comb }} \pm \sigma_{z_{\text {comb }}} \\
\text { (or redshift lower limit) }\end{array}$} & \multirow[t]{2}{*}{ Flag $^{\mathrm{b}}$} & \multicolumn{3}{|c|}{ NIR Blank Field Probability $P_{\text {blank }}(\%)^{\mathrm{c}}$} & \multicolumn{2}{|c|}{ rBCG Position } & \multirow[t]{2}{*}{ Imaging Ref. $^{\mathrm{d}}$} \\
\hline & R.A. (deg) & Decl. (deg) & & & & & NEWFIRM & Spitzer & WISE & R.A. (deg) & Decl. (deg) & \\
\hline SPT-CLJ2302-5225 & 345.6464 & -52.4329 & 4.60 & $\ldots$ & $>1.0$ & $\ldots$ & $\ldots$ & $\ldots$ & 83.3 & $\ldots$ & $\ldots$ & 1 \\
\hline SPT-CLJ2311-5011 & 347.8427 & -50.1838 & 4.64 & $\ldots$ & $>1.5$ & $\ldots$ & $\ldots$ & 38.4 & 69.7 & $\ldots$ & $\ldots$ & 1 \\
\hline SPT-CLJ2312-5820 & 348.0002 & -58.3419 & 4.78 & $\ldots$ & $0.83 \pm 0.05$ & 1 & $\ldots$ & 1.3 & 0.0 & 347.9912 & -58.3428 & 1 \\
\hline SPT-CLJ2329-5831 & 352.4760 & -58.5238 & 4.95 & $\ldots$ & $0.81 \pm 0.03$ & 1 & 2.3 & 0.0 & 0.1 & 352.4627 & -58.5128 & 1 \\
\hline SPT-CLJ2331-5051 & 352.9584 & -50.8641 & 8.04 & 0.576 & $0.61 \pm 0.02$ & 1 & .. & $\ldots$ & $\ldots$ & 352.9631 & -50.8650 & 2 \\
\hline SPT-CLJ2332-5358 & 353.1040 & -53.9733 & 7.30 & 0.402 & $0.38 \pm 0.02$ & 1 & $\ldots$ & $\ldots$ & $\ldots$ & 353.1144 & -53.9744 & 1,2 \\
\hline SPT-CLJ2334-5953 & 353.6989 & -59.8892 & 4.53 & $\ldots$ & $>1.5$ & $\ldots$ & $\ldots$ & 71.7 & 26.1 & $\ldots$ & $\ldots$ & 1 \\
\hline SPT-CLJ2337-5942 & 354.3544 & -59.7052 & 14.94 & 0.775 & $0.76 \pm 0.03$ & 1 & $\ldots$ & 0.3 & 0.0 & 354.3651 & -59.7013 & 2 \\
\hline SPT-CLJ2341-5119 & 355.2994 & -51.3328 & 9.65 & 1.003 & $0.93 \pm 0.07$ & 3 & $\ldots$ & 0.2 & 0.9 & 355.3015 & -51.3290 & 1,2 \\
\hline SPT-CLJ2342-5411 & 355.6903 & -54.1887 & 6.18 & 1.075 & $0.96 \pm 0.07$ & 3 & 7.4 & 2.4 & 9.6 & 355.6913 & -54.1848 & $1^{*}$ \\
\hline SPT-CLJ2343-5521 & 355.7574 & -55.3641 & 5.74 & $\ldots$ & $>1.5$ & $\ldots$ & $\ldots$ & 66.3 & 50.8 & $\ldots$ & $\ldots$ & 1,2 \\
\hline SPT-CLJ2343-5556 & 355.9290 & -55.9371 & 4.58 & $\ldots$ & $>1.2$ & $\ldots$ & 20.5 & $\ldots$ & 5.6 & $\ldots$ & $\ldots$ & $1^{*}$ \\
\hline SPT-CLJ2351-5452 & 357.8877 & -54.8753 & 4.89 & 0.384 & $0.37 \pm 0.02$ & 1 & $\ldots$ & $\ldots$ & $\ldots$ & 357.9086 & -54.8816 & $1^{*}$ \\
\hline SPT-CLJ2355-5056 & 358.9551 & -50.9367 & 5.89 & 0.320 & $0.28 \pm 0.02$ & 1 & $\ldots$ & $\ldots$ & $\ldots$ & 358.9477 & -50.9280 & 2 \\
\hline SPT-CLJ2359-5009 & 359.9208 & -50.1600 & 6.35 & 0.775 & $0.78 \pm 0.03$ & 1 & $\ldots$ & 0.0 & 0.3 & 359.9284 & -50.1672 & 2 \\
\hline
\end{tabular}

Notes.

${ }^{\text {a }}$ Spectroscopic redshift listed where available. Details on references and observations are given in Table 2.

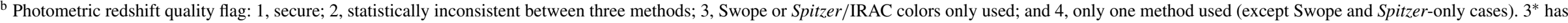
larger bias correction (SWOPE only photometric redshift (see the text for more detail).

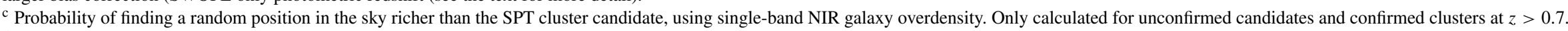

${ }^{\mathrm{d}}$ Cross-reference to imaging data. Only the deepest imaging data source is noted in this table. Internal references refer to Table 1 . Ref. $1^{*}$ indicates that BCS imaging data were used.

e Optical group on $\mathrm{N} z \sim 0.3$.

${ }^{\mathrm{f}}$ Optical only $z \sim 1.0$

$\mathrm{g}$ Very complex region, optical group on $\mathrm{NW} z \sim 0.4$, another group on $\mathrm{SW} z \sim 0.65$.

' Optical group within $1^{\prime}$ aperture $z \sim 0.35$.

${ }^{\mathrm{i}}$ Strong-lensing arc.

${ }^{j}$ Strong-lensing arc.

${ }^{\mathrm{k}}$ Optical group on $\mathrm{SW} z \sim 0.4$

${ }^{1}$ Optical group within $1^{\prime}$ aperture $z \sim 0.15$.

${ }^{\mathrm{m}}$ Optical group on $\mathrm{SE} z \sim 0.4$. 
In this work, we employ three methods (which we refer to as Method 1, 2, and 3 in the following sections) to estimate cluster redshifts from optical imaging data. Two methods (Method 1 and 2) use the color of the galaxies in the cluster red sequence, and the third (Method 3) uses the average of red-sequence galaxy photometric redshifts estimated with a neural-network algorithm, trained with the magnitudes of similar galaxies. In the optical analysis for our two previous cluster catalog releases (High et al. 2010; Williamson et al. 2011), we relied on Method 1 for the results and Method 3 as a cross-check. In this work, we improve the precision of the measured redshifts by applying multiple redshift estimation algorithms and combining the results. Through cross-checks during the analysis, we find that these methods have different failure modes and that comparing the results provides a way of identifying systems that require additional attention (including systems where the cluster's central region is contaminated by foreground stars or the cluster resides in a crowded field).

All three methods use the single-stellar-population models of Bruzual \& Charlot (2003, BC03 hereafter). These models allow us to transform the location of the red-sequence overdensity in color space to a redshift estimate. A model for the red galaxy population as a function of redshift is built assuming a single burst of star formation at $z_{f}=3$ followed by passive evolution thereafter. Models are selected over a range of metallicities and then calibrated to reproduce the color and tilt of the red sequence in the Coma cluster (Eisenhardt et al. 2007) at $z=0.023$. The calibration procedure is described in more detail in Song et al. (2012). The red-sequence model prescribed in a similar way has been demonstrated to adequately describe the bright end of the cluster red sequence (Blakeslee et al. 2003; Tran et al. 2007; Muzzin et al. 2009; High et al. 2010; Mancone et al. 2010; Stott et al. 2010; Song et al. 2012). These models are used in determining exposure times and appropriate filter combinations for imaging observations, and in the calculation of redshifts and redshift limits from those observations.

\subsubsection{Photometric Redshift Measurement Methods}

In Method 1, a cluster is confirmed by identifying an excess of galaxies with colors consistent with those derived from BC03 (simultaneously for all observed filters), after subtracting the background surface density. The background-subtracted galaxy number is extracted from an aperture within a radius of $(3.5,2.5,1.5)^{\prime}$ from the SPT candidate position and uses galaxies with photometric color uncertainties $\leqslant(0.25,0.35,0.45)$ and apparent magnitudes brighter than $m^{*}+(3,2,1)$ (or the magnitude limit of the data) in the red sequence based on the same BC03 models, for $z<0.2,0.2<z<0.6$, and $z>0.6$, respectively. The background measurement is obtained by applying the same criteria outside of the cluster search aperture. The redshift is estimated from the most significant peak in this red-sequence galaxy excess. Improvements over the implementation in $\mathrm{H} 10$ include using additional colors $(r-z$ and $g-i$, plus NIR colors) in the red-sequence fitting, using the deeper photometry available from co-added images, and sampling the entire CCD mosaic rather than a single CCD for better background estimation.

Method 2 is similar in that it searches for an overdensity of red-sequence galaxies. This method, used to estimate the redshifts for a sample of $46 \mathrm{X}$-ray-selected clusters (Šuhada et al. 2012), is described and tested in more detail in Song et al. (2012). It includes a measure of the background surface density based on the entire imaged sky area surrounding each cluster candidate and subtracts the background from the red galaxy counts in an aperture of $0.8 \mathrm{Mpc}$. Only galaxies with luminosity $>0.4 L^{*}$ and magnitude uncertainty $\leqslant 0.25$ are used, and the aperture and luminosity are recalculated for each potential redshift. Originally as described in Song et al. (2012), we search for an overdensity of red-sequence galaxies using two or three available color-redshift combinations simultaneously for every cluster; essentially, we scan outward in redshift using the following color combinations: $g-r$ and $g-i$ for $z<0.35$, $g-i$ and $r-i$ and $r-z$ for $0.3<z<0.75, r-z$ and $i-z$ for $z>0.75$. The cluster photometric redshift is extracted from the peak of the galaxy overdensity in redshift space. The redshift is then refined by fitting the red-sequence overdensity distribution in redshift space with a Gaussian function. The version used here (which is the same as the method used in Šuhada et al. 2012) has one more refinement, in which the colors of the galaxies that lie in the peak redshift bin identified by the overdensity method are converted into individual galaxy photometric redshifts. In this conversion we assume that the galaxies are red-sequence cluster member galaxies, and the photometric redshift uncertainty reflects the individual photometric color errors. A final cluster redshift is calculated as an inverse-variance-weighted mean of these galaxy photometric redshifts.

Method 3 shares the same principle as the other two in that it involves searching for a density peak in the galaxy distribution near the position of the SPT candidate. We first select individual red cluster members using location relative to the SPT candidate position and galaxy color as the criteria for cluster membership. For the redshifts presented here, this is done visually using pseudo-color co-added images for each cluster, although in principle this could be automated. Galaxy selection is not confined by a specific radial distance from SZ centers as in the other two methods, nor by photometric uncertainties. Selected galaxies are then fed into Artificial Neural Networks (ANNz; Collister \& Lahav 2004), which is trained using the same $\mathrm{BC} 03$ models used in the other methods. ANN $z$ returns redshift estimates for individual galaxies, and a peak in galaxy redshift distribution is adopted as the initial cluster redshift. Then, as in Method 2, individual galaxy photometric redshifts are averaged using inverse-variance weighting to produce the cluster photometric redshift. With this initial estimate of the redshift, we then perform an outlier rejection using iterative $1 \sigma$ clipping, where the $1 \sigma$ corresponds to the rms variation of the measured galaxy photometric redshift distribution. Once the rejection is carried out, we refine the cluster photometric redshift estimate using the weighted mean of the non-rejected sample of cluster galaxies. No outlier rejection is undertaken if there are fewer than 20 selected galaxies in the original sample.

Method 3 is a good cross-check, as well as a stand-alone redshift estimator, because we can visually confirm which galaxies contribute to the redshift determination. Although this method requires photometry in more than just two bands, it appears to be less susceptible to the problems in two-band methods that are associated with pileup of red-sequence galaxies at redshifts where the $4000 \AA$ break is transitioning out of a band.

Next, we characterize redshift estimates from each method using spectroscopically confirmed clusters. We use 47 clusters with spectroscopic redshifts $\left(z_{\text {spec }}\right)$ where only griz data are used for photometric redshift estimation. In this process, photometric redshift $\left(z_{\text {phot }}\right)$ biases (namely, smooth trends of photometric redshift offset as a function of redshift) are measured and corrected in Method 1 and 2, while no significant bias correction is necessary for Method 3. Bias corrections depend on several 


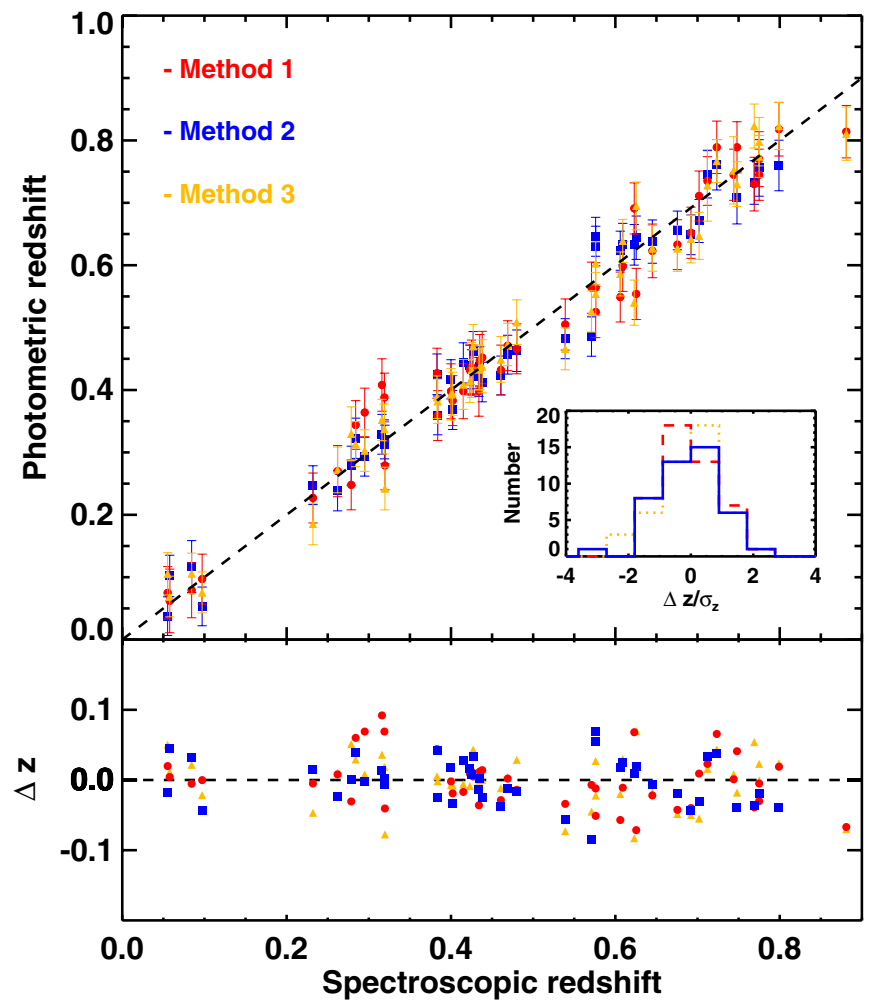

Figure 1. Top: photometric redshift $z_{\text {phot }}$ vs. spectroscopic redshift $z_{\text {spec }}$ for each redshift estimation method for 47 spectroscopically confirmed clusters at $z<0.9$ where we use only griz photometry. Bottom: the distribution of the photometric redshift residuals $\Delta z=z_{\text {phot }}-z_{\text {spec }}$ as a function of $z_{\text {spec }}$. Inset: the normalized residual distributions, which all have $\operatorname{rms}\left(\Delta z / \sigma_{z_{\text {phot }}}\right) \sim 1$. The rms scatter of $\Delta z /(1+z)$ is $0.028,0.023$, and 0.024 for Methods 1 (red dot and red dashed line), 2 (blue square and blue dash-dot-dot line), and 3 (yellow triangle and yellow dotted line), respectively.

(A color version of this figure is available in the online journal.)

factors, such as filters used for data, redshift of clusters, and the depth of the data. They are separately measured in those different cases per method as a function of $(1+z)$ at a level of 0.01-0.03 in redshift for clusters with redshift measured in griz filters, Spitzer-only, and $B V R I$ filters at $z>0.5$. The largest bias correction is needed for clusters observed from SWOPE using $B V R I$ filters with maximum correction of 0.13 at around $z \sim 0.4$ where the filter transitions from $B-V$ to $V-R$ occurs to capture the red-sequence population. This affects two clusters in the final sample. Once biases are removed, we examine the photometricto-spectroscopic redshift offsets to characterize the performance of each method. We find the rms in the quantity $\Delta z /\left(1+z_{\mathrm{spec}}\right)$, where $\Delta z=z_{\text {phot }}-z_{\text {spec }}$ have values of $0.028,0.023$, and 0.024 in Methods 1, 2, and 3, respectively (see Figure 1). We note that some of the bias and systematic error, especially at higher redshift, could be due to the mismatch between the spectral energy distributions (SEDs) in the red-sequence model and the cluster population, which could arise from variations in star formation history or active galactic nucleus (AGN) activity.

Our goal is not only to estimate accurate and precise cluster redshifts, but also to accurately characterize the uncertainty in these estimates. To this end, we use the spectroscopic subsample of clusters to estimate a systematic floor $\sigma_{\text {sys }}$ in addition to the statistical component. We do this by requiring that the reduced $\chi^{2}$ describing the normalized photometric redshift deviations from the true redshifts $\chi_{\text {red }}^{2}=\sum\left(\Delta z / \sigma_{z_{\text {phot }}}\right)^{2} / N_{\text {dof }}$ have a value of $\chi_{\text {red }}^{2} \sim 1$ for each method, where $\sigma_{z_{\text {phot }}}$ is the uncertainty in measured $z_{\text {phot }}$ and $N_{\text {dof }}$ is the number of degrees of freedom. We adopt uncertainties $\sigma_{z \text { phot }}^{2}=\sigma_{\text {stat }}^{2}+\sigma_{\text {sys }}^{2}$ and adjust $\sigma_{\text {sys }}$ to obtain the correct $\chi_{\text {red }}^{2}$. In this tuning process we also include redshift estimates of the same cluster from multiple instruments when that cluster has been observed multiple times. This allows us to test the performance of our uncertainties over a broader range of observing modes and depths than is possible if we just use the best available data for each cluster.

For Method 1, we separately measure the systematic floor $\sigma_{\text {sys }}$ for each different photometric band set. For the griz $K_{s}$ instruments (Megacam, IMACS, LDSS3, MOSAIC2, NEWFIRM), we estimate $\sigma_{\text {sys }}=0.039$; for the BVRI instrument (Swope), $\sigma_{\text {sys }}=0.033$; and for Spitzer-only, $\sigma_{\text {sys }}=0.070$. In Method 2 , we find $\sigma_{\text {sys }}=0.030$ for the griz instruments (Megacam, IMACS, LDSS3, MOSAIC2). For Method 3 we estimate $\sigma_{\text {sys }}=0.028$ for the griz instruments.

Once this individual estimation and calibration is done, we conduct an additional test on the redshift estimation methods, again using the spectroscopic subsample. The purpose of this test is to see how the quality of photometry (i.e., follow-up depth) affects the estimations. We divide the spectroscopic sample into two groups: in one group, the photometric data are kept at full depth, while the photometric data in the other group are manually degraded to resemble the data from the shallowest observations in the total follow-up sample. To create the "shallow" catalogs, we add white noise to the full-depth co-adds and then extract and calibrate catalogs from these artificially noisier images. Results of this test show that the accuracy of the photometric redshift estimation is affected by the poorer photometry, but that this trend is captured well by the statistical uncertainties in each estimation method.

\subsubsection{Combining Photo-z Estimates to Obtain $z_{\mathrm{comb}}$}

Once redshifts and redshift uncertainties are estimated with each method independently, we compare the different redshift estimates of the same cluster. Note that this comparison is not possible for Swope or Spitzer-only redshifts, which are measured only with Method 1. Outliers at $\geqslant 3 \sigma(>6 \%)$ in $1+z_{\text {phot }}$ are identified for additional inspection. In some cases, there is an easily identifiable and correctable issue with one of the methods, such as misidentification of cluster members. If, however, it is not possible to identify an obvious problem, the outliers are excluded from the combining procedure. This outlier rejection, which occurs only in two cases, causes less than 0.05 change in the combined $z_{\text {phot }}$ in both cases.

We combine the individual estimates into a final best redshift estimate, $z_{\mathrm{comb}}$, using inverse-variance weighting and accounting for the covariance between the methods, which we expect to be non-zero given the similarities in the methods and the common data used. Correlation coefficients for the photometric redshift errors among the different methods are measured using the spectroscopic sample. The measured correlation coefficient, $r_{i j}$, between each pair of methods is 0.11 (Method 1 and 2), 0.40 (Method 2 and 3), and 0.19 (Method 1 and 3).

With the correlation coefficients we construct the optimal combination of the individual estimates as

$$
z_{\mathrm{comb}}=\frac{1}{\sum_{i j} W_{i j}} \sum_{i} \sum_{j} W_{i j} z_{j},
$$

where $W_{i j}=C_{i j}^{-1}$, and the covariance matrix $C_{i j}$ is comprised of the square of the individual uncertainties along the diagonal 


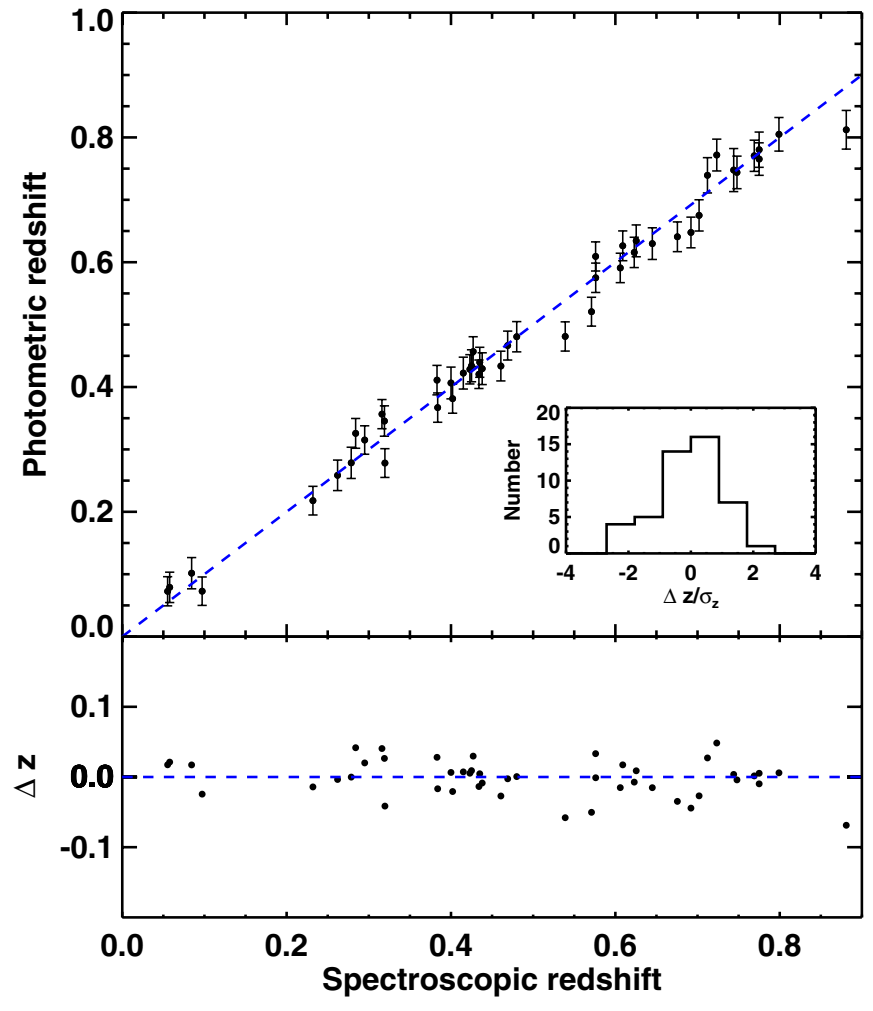

Figure 2. Top: weighted mean photometric redshift $z_{\text {comb }}$ vs. spectroscopic redshift using the same subsample as in Figure 1. Bottom: the distribution of the redshift errors. The rms scatter in $\Delta z /\left(1+z_{\text {spec }}\right)=0.017$. Inset: histogram of the normalized redshift error distribution, which is roughly Gaussian with $\mathrm{rms} \simeq 1$.

(A color version of this figure is available in the online journal.)

elements $\left(\sigma_{i}^{2}\right)$ and the product of the measured correlation coefficient and the two individual uncertainty components (i.e., $r_{i j} \sigma_{i} \sigma_{j}$ ) on the off-diagonal elements. The associated uncertainty is

$$
\sigma_{z_{\mathrm{comb}}}^{2}=\frac{1}{\sum_{i j} W_{i j}} .
$$

Because of the positive correlations between the three methods' errors, the errors on $z_{\text {comb }}$ are larger than would be the case for combining three independent estimates; however, we do see an improvement in the performance of the combined redshifts relative to the individual estimates that is consistent with the expectation given the correlations. The performance of this combined redshift method is presented in Figure 2; the residual distribution is roughly Gaussian, and the associated uncertainties provide a good description of the scatter of the redshift estimates about the spectroscopic redshifts (the rms variation of $\Delta z / \sigma_{z_{\text {phot }}}$ is 1.04). The benefit from combining different measurements is evidenced from the tighter distribution in the redshift versus $z_{\text {spec }}$ plot; the rms scatter of $\Delta z /\left(1+z_{\text {spec }}\right)$ is 0.017 , corresponding to a $\sim 40 \%$ improvement in the accuracy relative to the accuracy of a single method.

\subsubsection{Spitzer Photometric Redshifts}

For clusters where we do not have deep enough optical data to estimate a redshift but that do have Spitzer coverage, we use the algorithm used in Method 1 to measure the redshifts using Spitzer-only colors in the same manner as we do with optical data. Overdensities of red galaxies in clusters have been identified using Spitzer-only color selection at high redshift, where

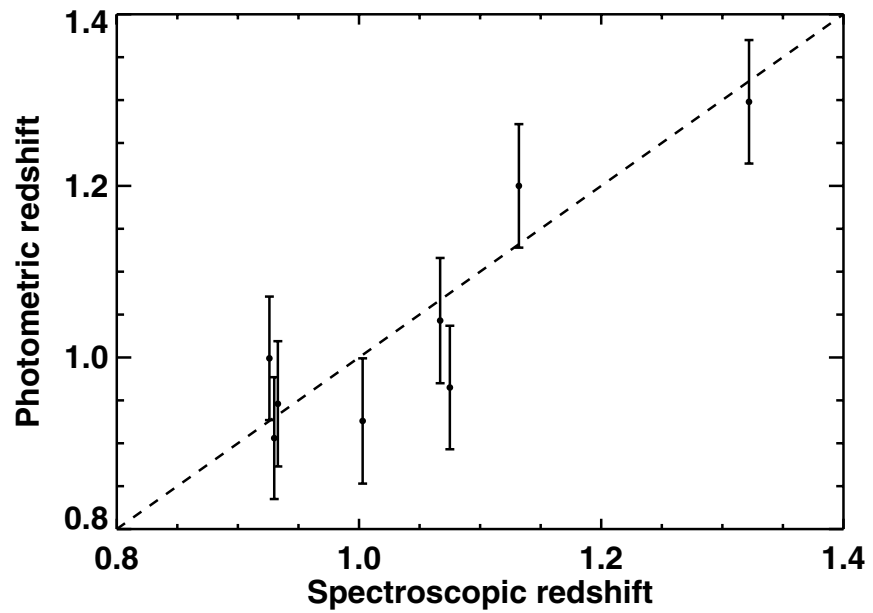

Figure 3. Photometric redshift vs. $z_{\text {spec }}$ for clusters where only the Spitzer/IRAC 3.6-4.5 $\mu \mathrm{m}$ colors are used. In all cases where we present Spitzer photo- $z$ 's, we have optical data to rule out the presence of a low-redshift cluster.

the IRAC bands are probing the peak of the stellar emission (Stern et al. 2005; Papovich 2008), rather than bracketing the $4000 \AA$ break. Note that the concerns about the impact of recent star formation or AGN activity on photometric redshift estimates are not as serious in the IRAC bands as in the optical bands, because the portion of the spectrum probed is less sensitive to these potential sources of contamination. In our sample, the comparison of Spitzer-only redshifts with spectroscopically derived redshifts shows good performance, indicating that the assumption of a well-developed red sequence appears to hold out to $z \gtrsim 1$ (e.g., Bower et al. 1992; Eisenhardt et al. 2008; Muzzin et al. 2009). Note that the possibility of the cluster being at lower redshift is already ruled out from the available optical data for these candidates.

Figure 3 shows the performance of the Spitzer-only redshifts in eight clusters where spectroscopic redshifts are available. Although the accuracy in $z_{\text {phot }}$ is lower $(\Delta z /(1+z) \approx 0.049)$ than those derived from optical-only or optical-IRAC colors, the performance is reliable. We flag these cases in the final table to make note of this difference in method. The larger uncertainties of Spitzer-only derived redshifts are possibly due to the broad width of IRAC filters and the fact that AGN emission or vigorous star formation can shift the location of the $1.6 \mu \mathrm{m}$ bump.

\subsubsection{Redshift Limits}

In most cases there is an obvious, rich overdensity of red cluster galaxies in our follow-up imaging, from which it is straightforward to confirm the galaxy cluster giving rise to the SZ signal and to estimate the cluster redshift. For unconfirmed candidates, it is not possible to say with absolute certainty that no optical/NIR counterpart exists; with real, finite-depth optical and NIR data, the possibility always exists that the cluster is distant enough that no counterpart would have been detected at the achieved optical/NIR depth. Assigning a relative probability to these two interpretations of an optical/NIR non-detection (i.e., a false detection in the SZ data or a higher-redshift cluster than the optical/NIR observations could detect) is especially important for interpreting the SZ cluster sample cosmologically. To this end, we calculate a lower redshift limit for every SZ-selected candidate for which no counterpart has been found. Because the optical/NIR follow-up data are not homogeneous, we do this separately for each unconfirmed candidate. 
To estimate the depth of the optical/NIR co-added images, we utilize a Monte Carlo based technique described in Ashby et al. (2009). In brief, we perform photometry of the sky in various apertures at 1500 random positions in each image. To measure the sky noise, we then fit a Gaussian function to the resulting flux distribution (excluding the bright tail which is biased by real sources in the image). Taking the measured sky noise from $3^{\prime \prime}$-diameter apertures, we add a PSF-dependent aperture correction. A redshift limit is derived for each filter by matching a $0.4 L^{*}$ red-sequence galaxy from the BC03 model to the measured $10 \sigma$ magnitude limit. We use the redshift limit from the second deepest filter with regards to $0.4 L^{*}$ redsequence objects, as we require a minimum of two filters to measure a redshift. These redshift limits are compared to limits derived by comparing observed number counts of galaxies as a function of magnitude to distributions derived from much deeper data (Zenteno et al. 2011). We find the two independent redshift limit estimations are in good agreement.

For cluster candidates with Spitzer/IRAC observations, the redshift estimation is not limited by the depth of the optical data, and we use the IRAC data to calculate a lower redshift limit for these candidates. The IRAC data are highly uniform, with depth sufficient to extract robust photometry down to $0.1 L^{*}$ out to a redshift of $z=1.5$. In principle, $\sim 0.5 L^{*}$ photometry should be sufficient for redshift estimation; however, we adopt $z=1.5$ as a conservative lower redshift limit for any unconfirmed candidates with IRAC data.

\subsection{NIR Overdensity Estimates for Unconfirmed Candidates}

For cluster candidates for which we are unable to estimate a redshift, we can in principle go beyond a simple binary statement of "confirmed/unconfirmed" using NIR data. Even if there is not a sufficient number of galaxies in the NIR data to estimate a red sequence, there is information in the simple overdensity of objects (identified in a single NIR band) within a certain radius of an SPT candidate, and we can use this information to estimate the probability of that candidate being a real, massive cluster. We can then use this estimate to sharpen our estimate of the purity of the SPT-selected cluster sample. We calculate the single-band NIR overdensity for all unconfirmed candidates using WISE data, and we compare that value to the same statistic estimated on blank-field data. We perform the same procedure using Spitzer/IRAC and NEWFIRM data for unconfirmed candidates that were targeted with those instruments. For comparison, we also calculate the same set of statistics for each confirmed cluster above $z=0.7$.

We estimate the galaxy overdensity within a $1 \operatorname{arcmin}^{2}$ aperture. To increase the signal to noise of the estimator, we assume an angular profile shape for the cluster galaxy distribution and fit the observed distribution to this shape. The assumed galaxy density profile is a projected $\beta$ model with $\beta=1$ (the same profile assumed for the SZ signal in the matched-filter cluster detection algorithm in R12). We have tried using a projected Navarro-Frenk-White profile as well, and the results do not change in any significant way (due to the relatively low signal to noise in the NIR data). The central amplitude, background amplitude, scale radius, and center position (with respect to the center of the SZ signal in SPT data) are free parameters in the fit. The number of galaxies above background within $1 \operatorname{arcmin}^{2}$ - which we will call $\Sigma_{1^{\prime}}$-is then calculated from the best-fit profile. The same procedure is repeated on fields not expected to contain massive galaxy clusters, and the value of
$\Sigma_{1^{\prime}}$ for every SPT candidate is compared to the distribution of $\Sigma_{1^{\prime}}$ values in the blank fields. The key statistic is the fraction of blank fields that had a $\Sigma_{1^{\prime}}$ value larger than a given SPT candidate, and that value is recorded as $P_{\text {blank }}$ in Table 3 for every high-redshift $(z \geqslant 0.7)$ or unconfirmed candidate. This technique, including using the blank-field statistic as the primary result, is similar to the analysis of WISE data in the direction of unconfirmed Planck Early SZ clusters in Sayers et al. (2012), although that analysis used raw galaxy counts within an aperture rather than profile fitting.

The model fitting is performed using a simplex-based $\chi^{2}$ minimization, with any parameter priors enforced by adding a $\chi^{2}$ penalty. The positional offset $\chi^{2}$ penalty is $\Delta \chi^{2}=$ $\left(\Delta \theta / \sigma_{\Delta \theta}\right)^{2}$, where $\sigma_{\Delta \theta}$ is chosen to be 0.25 , based on the SZ/BCG offset distribution in Figure 7 and the value of $r_{200}$ for a typical-mass SPT cluster at high redshift. ${ }^{41}$ A prior is enforced on the scale radius from below and above by adding $\chi^{2}$ penalties of $\left(\theta_{s} / \theta_{s, \max }\right)^{2}$ and $\left(\theta_{s, \min } / \theta_{s}\right)^{2}$, where $\theta_{s, \max }$ is chosen to be 0.75 based on the $\theta_{s}$ distribution in known high-redshift SPT clusters with NIR data, and $\theta_{s, \text { min }}$ is chosen to be 0.125 to prevent the fitter from latching onto small-scale noise peaks.

For Spitzer/IRAC and WISE, the fit is performed on the 3.6 $\mu \mathrm{m}$ and the $3.4 \mu \mathrm{m}$ data, respectively; for NEWFIRM, the fit is performed on the $K_{s}$-band data. For both Spitzer/IRAC and NEWFIRM, a single magnitude threshold is used for every candidate; this threshold is determined by maximizing the signal to noise on the $\Sigma_{1^{\prime}}$ estimator on known clusters while staying safely away from the magnitude limit of the shallowest observations. The Spitzer/IRAC data are very uniform, and the $3.6 \mu \mathrm{m}$ magnitude threshold chosen is 18.5 (Vega). The NEWFIRM $K_{s}$-band data are less uniform, but a magnitude threshold of 18 (Vega) is safe for all observations. For these instruments (IRAC and NEWFIRM), the blank fields on which the fit is performed come from the Spitzer Deep Wide-Field Survey (SDWFS) region (Ashby et al. 2009), which corresponds to the Bootes field of the NOAO Deep Wide-Field Survey (NDWFS; Jannuzi \& Dey 1999). The depth of the SDWFS/ NDWFS observations for both instruments (19.8 in Vega for Spitzer/IRAC $3.6 \mu \mathrm{m}$ and 19.5 in Vega for NEWFIRM $K_{s}$ ) is more than sufficient for our chosen magnitude thresholds.

For WISE, in which the non-uniform sky coverage results in significant variation in magnitude limits, we perform the blankfield fit on data in the immediate area of the cluster (within a $\sim 20^{\prime}$ radius). Under the assumption that the WISE magnitude limit does not vary over this small an angular scale, we use all detected galaxies brighter than 18th magnitude (Vega) in both the cluster and blank-field fits.

\subsection{Identifying rBCGs in SPT Clusters}

An $\mathrm{rBCG}$ in this work is defined as the brightest galaxy among the red-sequence galaxies for each cluster. We employ the terminology $\mathrm{rBCG}$, rather than BCGs, to allow for the rare possibility of an even brighter galaxy with significant amounts of ongoing star formation, because the selection is restricted by galaxy colors along the cluster red sequence. We visually inspect pseudo-color images built with the appropriate filter combinations (given the cluster redshift) around the SZ candidate position. We search a region corresponding to the projected cluster virial region, defined by $\theta_{200}$, given the mass

\footnotetext{
$41 r_{200}$ is defined as the radius within which the average density is 200 times the mean matter density in the universe.
} 
estimate from the observed cluster SPT significance $\xi$ and photometric redshift $z_{\text {phot }}$.

There are 12 clusters out of the 158 with measured photometric redshifts (excluding the candidates with redshift limits) that are excluded from the rBCG selections. Eight of those are excluded due to contamination by a bright star that obscures more than one-third of the area of the $3 \sigma$ SPT positional uncertainty region. Another cluster is excluded due to a bleed trail making the rBCG selection ambiguous, and three other clusters are excluded due to a high density in the galaxy population that, given the delivered image quality, makes it impossible to select the $\mathrm{rBCG}$.

\section{RESULTS}

The complete list of 224 SPT cluster candidates with SZ detection significance $\xi \geqslant 4.5$ appears in Table 3 . The table includes SZ cluster candidate positions on the sky (R.A., Decl.), SZ detection significance $(\xi)$, and spectroscopic redshift $\left(z_{\mathrm{spec}}\right)$ when available. For confirmed clusters, the table includes photometric redshift and uncertainty $\left(z_{\text {comb }} \pm \sigma_{z_{\text {comb }}}\right)$, estimated as described in Section 3.1. Unconfirmed candidates are assigned redshift lower limits, estimated as described in Section 3.1.4.

We also report a redshift quality flag for each $z_{\text {phot }}$ in Table 3. For most of the confirmed clusters with reliable photometric redshift measurements, we set Flag $=1$. There is one cluster (SPT-CL J2146-4846) for which the three individual photometric redshifts are not statistically consistent ( $\gtrsim 3 \sigma$ outliers) for which we set Flag $=2$. We still report the combined redshift for that cluster as in other secure systems. We have 6 cases where we only use Swope + Method 1, and 25 cases where we only use Spitzer + Method 1 for redshift estimation, both cases marked with Flag $=3$. We note that the photometric redshift bias correction for two clusters (SPT-CL J0333-5842 and SPT-CL J0456-6141) is at a higher level than the typical bias correction on other clusters (see Section 3.1.1 for more detail on the bias correction. There are two cases (SPT-CL J0556-5403 and SPT-CL J0430-6251) where we quote only a Method 1 redshift even for MOSAIC or IMACS data, marked with Flag $=4$. In the co-added optical images for SPT-CL J05565403 , we identify an overdensity of faint red galaxies at the location of the SPT candidate. These optical data are too shallow, however, to allow for secure redshift estimation, but we are able to measure a redshift by combining this data with NEWFIRM imaging. This cluster is the only candidate where we rely on photometric redshift from $i-K_{s}$. SPT-CL J0430-6251 is in a field very crowded with large-scale structure, making redshift estimation difficult.

In the Appendix, we discuss certain individually notable candidates-such as associations with known clusters that appear to be random superpositions and candidates with no optical/NIR confirmation but strong evidence from the NIR overdensity statistic.

\subsection{Redshift Distribution}

The redshift distribution for the 158 confirmed clusters is shown in Figure 4. The median redshift is $z=0.57$, with 28 systems ( $\sim 18 \%$ of the sample) lying at $z>0.8$. The cluster with the highest photometric redshift is SPT-CL J2040-4451 at $z=1.35 \pm 0.07$ (estimated using Spitzer/IRAC data) and the highest-redshift spectroscopically confirmed cluster is SPT-CL J0205-5829 at $z=1.32$. (This cluster is discussed in detail in Stalder et al. 2012.)

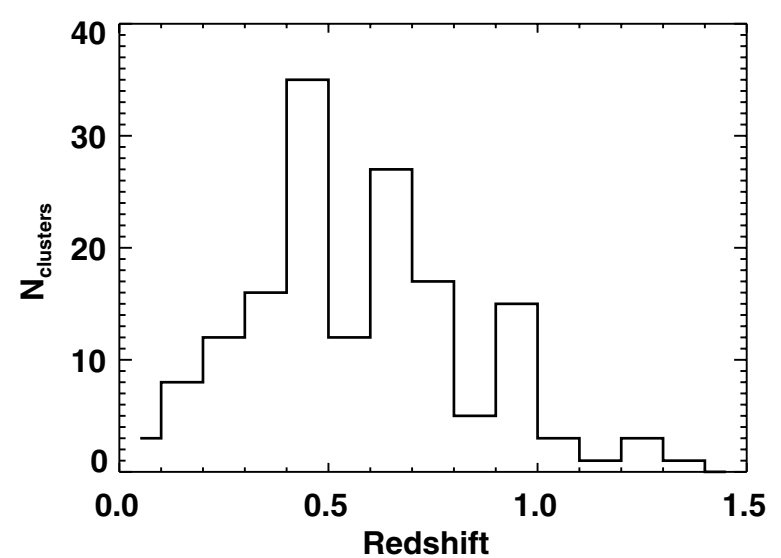

Figure 4. Redshift histogram of 158 confirmed clusters, in bins of $\Delta z=0.1$. Note that about $18 \%$ of the total sample comes from $z \geqslant 0.8$.

The high fraction of SPT clusters at $z>0.8$ is a consequence of the redshift independence of the SZ surface brightness and the arcminute angular resolution of the SPT, which is well matched to the angular size of high- $z$ clusters. X-ray surveys, in contrast, are highly efficient at finding nearby clusters, but the mass limit of an X-ray survey will increase with redshift due to cosmological dimming. ROSAT cluster surveys lack the sensitivity to push to these high redshifts except in the deepest archival exposures. XMM-Newton archival surveys (e.g., LloydDavies et al. 2011; Fassbender et al. 2011) and coordinated surveys of contiguous regions (e.g., Pacaud et al. 2007; Šuhada et al. 2012) have sufficient sensitivity to detect systems like those found by SPT, but the solid angle surveyed is currently smaller. For example, the Fassbender et al. (2011) survey for highredshift clusters will eventually cover approximately $80 \mathrm{deg}^{2}$, whereas the mean sky density of the SPT high-redshift and high-mass systems is around one every $25 \mathrm{deg}^{2}$. Therefore, one would have expected the Fassbender et al. (2011) XDCP survey to have found around three clusters of comparable mass to the SPT clusters, which is in fact consistent with their findings. The vast majority of the high-redshift X-ray-selected sample available today is of significantly lower mass than SPT-selected samples, simply because the X-ray surveys do not yet cover adequate solid angle to find these rare, high-mass systems.

Clusters samples built from NIR galaxy catalogs have an even higher fraction of high-redshift systems than SZ-selected samples-for example, in the IRAC Shallow Cluster Survey (ISCS; Eisenhardt et al. 2008) a sample of 335 clusters has been identified out to $z \sim 2$, a third of which are at $z>1$. However, the typical ISCS cluster mass is $\sim 10^{14} M_{\odot}$ (Brodwin et al. 2007), significantly lower than the minimum mass of the SPT high-redshift sample. As with the X-ray-selected samples, the Spitzer sample includes some massive clusters, including the recently discovered IDCS J1426.5+3508 at $z=1.75$, which was subsequently also detected in the SZ (Stanford et al. 2012; Brodwin et al. 2012). However, the Spitzer surveys to date do not cover the required solid angle to find these massive systems in the numbers being discovered by SPT.

\subsection{Purity of the SPT Cluster Candidates}

For a cluster sample to be useful for cosmological purposes, it is important to know the purity of the sample, defined as

$$
f_{\text {pure }}=\frac{N_{\text {real }}}{N_{\text {tot }}}=1-\frac{N_{\text {false }}}{N_{\text {tot }}},
$$




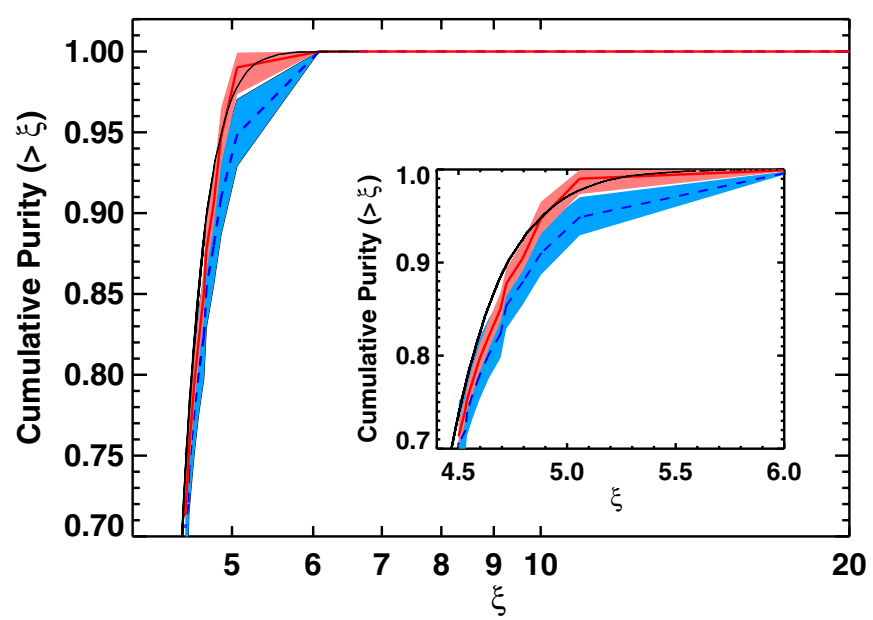

Figure 5. Cumulative purity estimates derived from the optical/NIR followup compared to simulated purity predictions (solid black line). The inset plot is zoomed-in to the $\xi$ range between 4.5 and 6.0 and binned more finely. The purity is calculated from the follow-up confirmation rate: (1) (dashed blue) assuming all clusters without a clear optical or NIR counterpart are false SZ detections (i.e., $100 \%$ optical completeness) and all optical confirmations are robust (100\% optical purity); and (2) (dash-dot-dot red) assuming, as justified in the text, 97\% optical completeness and $96 \%$ optical purity but taking into account clusters confirmed through other means such as X-ray observations. $1 \sigma$ uncertainties in the purity estimates from follow-up are shown with shaded blue or red regions (see Section 4.2).

(A color version of this figure is available in the online journal.)

where $N_{\text {tot }}$ is the total number of cluster candidates, $N_{\text {real }}$ is the number of candidates corresponding to real clusters, and $N_{\text {false }}$ is the number of false detections. For an SZ-selected cluster sample with reasonably deep and complete optical/NIR follow-up, a first-order estimate of $N_{\text {real }}$ is simply the number of candidates with successfully estimated redshifts. In Figure 5, we show two estimations of purity for the $720 \mathrm{deg}^{2}$ SPT-SZ sample; the first in blue, assuming that all cluster candidates with no redshift measurements are noise fluctuations, and the other in red, taking into account incompleteness of our followup data. The blue/red shaded regions in the figure correspond to the $1 \sigma$ uncertainties on the purity, estimated from Poisson noise on $N_{\text {false }}$ for the blue region and as described below for the red region. We also show the expected purity, estimated from the total number of candidates in the sample presented in this work combined with the false detection rate from the simulations used to test the SZ cluster finder (R12, Figure 1).

The possibility of real clusters beyond the redshift reach of our optical/NIR redshift estimation techniques makes the blue line in Figure 5 a lower limit to the true purity of the sample. As discussed in Section 3.2, we use single-band NIR data to estimate the probability that each unconfirmed candidate is a "blank field," i.e., a field with typical or lower-thantypical NIR galaxy density. Candidates with no optical/NIR confirmation but with a low blank-field probability $P_{\text {blank }}$, are potential high-redshift systems that merit further followup study. These systems can also give an indication of how much we underestimate our sample purity when we assume any optical/NIR non-confirmation is a spurious SPT detection. By definition, a low $P_{\text {blank }}$ implies some NIR overdensity toward the SPT detection, but perhaps not large enough to be an SPT-detectable cluster. We can roughly calibrate the $P_{\text {blank }}$ values to SPT detectability by investigating the results of the NIR overdensity estimator on solidly confirmed, high-redshift SPT clusters. There are 19 clusters with spectroscopic redshifts above $z=0.7$, and the average Spitzer/IRAC $P_{\text {blank }}$ value for these clusters is 0.04 , while the average WISE $P_{\text {blank }}$ value is 0.05 . Only three of these clusters have NEWFIRM data, and the average NEWFIRM $P_{\text {blank }}$ value is 0.07 . Only one cluster in this high- $z$ spectroscopic sample has an IRAC $P_{\text {blank }}>0.1$, while three have WISE $P_{\text {blank }}>0.1$. So a rough threshold for SPT-type clusters appears to be $P_{\text {blank }} \leqslant 0.1$. We have nine unconfirmed cluster candidates that meet this criterion in at least one of the NIR catalogs, including five that are at $P_{\text {blank }} \leqslant 0.05$. If we assumed that all of the $P_{\text {blank }} \leqslant 0.05$ clusters were real, it would imply that the completeness of the optical/NIR redshift estimation was $\sim 97 \%$, i.e., we have 163 real clusters of which we were able to estimate redshifts for 158 .

Conversely, the possibility of false associations of spurious SZ detections with optical/NIR overdensities would act in the other direction. Tests of one of the red-sequence methods on blank-field data produced a significant red-sequence detection on approximately $4 \%$ of fields without SPT detected clusters. Assuming that the cross-checks with other methods would remove some of these, we can take this as an upper limit to this effect.

We therefore provide a second estimate of purity from the optical/NIR confirmation rate, taking into account the possibility of real clusters for which we were unable to successfully estimate a redshift (redshift completeness <100\%) and spurious optical/NIR associations with SPT noise peaks (redshift purity $<100 \%$ ). From the above arguments, we assume $97 \%$ for redshift completeness and $96 \%$ for redshift purity. For each value of SPT significance $\xi$, we use binomial statistics to ask how often a sample of a given purity with total candidates $N(>\xi)$ would produce the observed number of successful optical/NIR redshift estimates $N_{\text {conf }}(>\xi)$, given the redshift completeness and false rate. An extra constraint is added to this calculation based on data independent of the optical/NIR imaging that confirms many of the SPT candidates as real, massive galaxy clusters. Specifically, we assume an SPT candidate is a real, massive cluster independent of the optical/NIR imaging data (and remove the possibility of that candidate being a false optical/NIR confirmation of an SPT noise peak) if: (1) it is associated with a ROSAT Bright Source Catalog source; (2) we have obtained $\mathrm{X}$-ray data in which we can confirm a strong, extended source; or (3) we have obtained spectroscopic data and measured a velocity dispersion for that system. The red solid line and shaded region in Figure 5 show the maximum-likelihood value and $68 \%$ limits for the true purity of the SPT sample under these assumptions.

The purity measured in this work is in good agreement with the model for the SPT sample purity. In particular, all clusters with $\xi>6$ have identified optical counterparts with photometric redshift estimates. This is consistent with the expectation of the model and a demonstration that the SPT-selected galaxy cluster sample is effectively uncontaminated at $\xi>6$. With decreasing significance, the number of noise fluctuations in the SPT maps increases compared to the number of real clusters on the sky, and the purity decreases. The cumulative purity of the sample is $\sim 70 \%$ above $\xi=4.5$ and reaches $\sim 100 \%$ above $\xi=5.9$. Of course, if one requires optical confirmation in addition to the SPT detection, then the sample is effectively $100 \%$ pure over the full sample at $\xi>4.5$.

We note that there is no significant difference in false detection rate (based on optical confirmation alone) between cluster candidates selected with $150 \mathrm{GHz}$ data alone and those detected with the multiband strategy (see Section 2.1 for details). Roughly one-fourth of the survey area was searched 


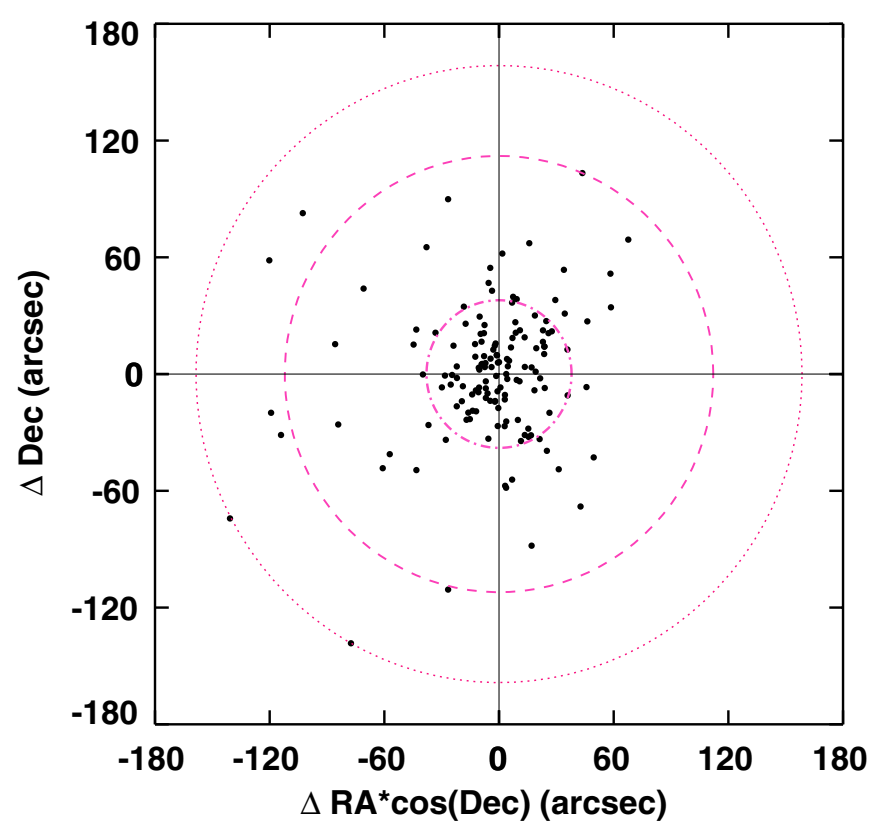

Figure 6. rBCG positions are plotted as offsets from SPT candidate positions for 146 systems with clearly defined rBCGs. The magenta concentric circles enclose $68 \%$ (dash-dotted line), 95\% (dashed line), and 99\% (dotted line) of the whole rBCG sample and have radii of $38^{\prime \prime} .0,112^{\prime \prime} .1$, and $158^{\prime \prime} .6$.

(A color version of this figure is available in the online journal.)

for clusters using $150 \mathrm{GHz}$ data only, and in that area we have 12 unconfirmed candidates, including one above $\xi=5$; in the three-fourths of the area selected using multiband data, we have 54 unconfirmed candidates, including 5 above $\xi=5$. These totals are consistent within $1 \sigma$ Poisson uncertainties.

The high purity of the SPT-selected cluster sample is comparable to the purity obtained in previous X-ray cluster surveys (i.e., Vikhlinin et al. 1998; Mantz et al. 2008; Vikhlinin et al. 2009), indicating that these intracluster-medium-based selection techniques, when coupled with optical follow-up, provide a reliable way to select clean samples of clusters for cosmological analysis.

\section{3. rBCG Offsets in SPT Clusters}

The position of the rBCG in galaxy clusters is a property of interest for both astrophysical and cosmological cluster studies, as it is a possible indication of a cluster's dynamical state. In relaxed clusters, it is expected that dynamical friction will tend to drive the most massive galaxies to the bottom of the cluster potential well, which would coincide with the centroid of the $\mathrm{X}$-ray and SZ signatures. On the other hand, in cases of merging systems one would expect two different rBCGs, and one or both could appear well separated from the X-ray or SZ centroid. Several studies have shown a tight correlation between the X-ray centroid and the rBCG position (Lin \& Mohr 2004; Haarsma et al. 2010; Mann \& Ebeling 2012; Stott et al. 2012), although Fassbender et al. (2011) provide evidence that at high redshift the BCG distribution is less centrally peaked. Here we examine the rBCG positions with respect to the centroid of the SZ signal in the SPT cluster sample.

The position of each rBCG is listed in Table 3, and the offsets from the SZ centroids in arcsec are plotted in Figure 6. The rings correspond to different fractions of the full population of clusters: $68 \%, 95 \%$, and $99 \%$. These rings have radii of $38^{\prime \prime} 0,112^{\prime \prime} .1$, and $158^{\prime \prime} 6$, respectively. The $\mathrm{rBCG}$ population is

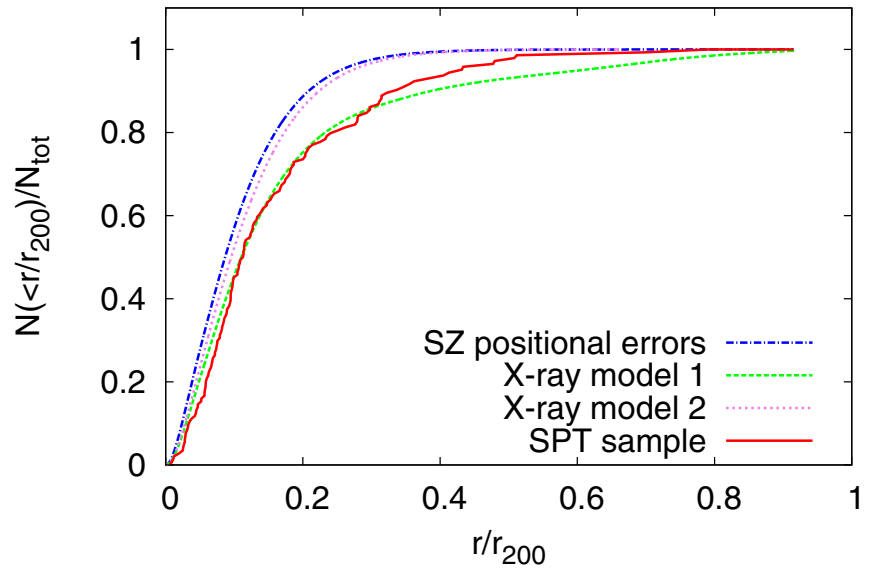

Figure 7. Normalized cumulative distribution of rBCG offsets from SPT candidate positions as a function of $r / r_{200}$ for the SPT cluster sample (red solid line), the expected distribution given SPT positional uncertainties if all $\mathrm{rBCG}$ were at exactly the center of the true SZ centroid (blue dash-dotted line), and the expected distribution given SPT positional uncertainties if the underlying distribution of offsets matched those of two different X-ray-selected cluster samples (green dashed line; Lin \& Mohr 2004) and (magenta dotted line; Mann \& Ebeling 2012). The K-S probability that the observed rBCG distribution and the SPT positional error distribution are drawn from the same parent distribution is $0.09 \%$, but the observed distribution is statistically consistent with the distribution from the X-ray-selected sample convolved with the SPT positional uncertainty distribution. There is no evidence in the $\mathrm{rBCG}$ offset distribution that SPT-selected clusters are more merger-rich than X-ray-selected clusters.

(A color version of this figure is available in the online journal.)

centrally concentrated with the bulk of the SPT-selected clusters having rBCGs lying within about $1^{\prime}$ of the candidate position.

Given the broad redshift range of the cluster sample, the $\mathrm{rBCG}$ distribution in cluster coordinates $r / r_{200}$ is more physically interesting. We use the cluster redshifts from this work and the SZ-derived masses from R12 to calculate $r_{200}$ for each cluster. The red line in Figure 7 is the cumulative distribution of the rBCGs as a function of $r / r_{200}$. In this distribution, $68 \%$ of the rBCGs lies within $0.17 r_{200}, 95 \%$ within $0.43 r_{200}$, and $99 \%$ within $0.70 r_{200}$.

We check for any effects of mm-wave selection and redshift estimation on the rBCG offset distribution by splitting the sample three ways: (1) clusters selected using $150 \mathrm{GHz}$ data only versus clusters selected using multiband data; (2) clusters with spectroscopic redshifts versus clusters with photometric redshifts only; (3) clusters with secure photometric redshifts $($ Flag $=1)$ versus clusters with flagged redshifts (Flag $>1$, see Section 4 for details). We see no evidence that the rBCG offsets (in units of $r_{200}$ ) in these subsamples are statistically different. A Kolmogorov-Smirnov (K-S) test results in probabilities of $84 \%, 39 \%$, and $34 \%$ that these respective subsamples are drawn from the same underlying distribution.

We investigate the importance of the SPT candidate positional uncertainty by modeling the expected radial distribution in the case where all rBCGs are located exactly at the cluster center. The $1 \sigma$ SPT positional uncertainty for a cluster with a pressure profile given by a spherical $\beta$ model with $\beta=1$ and scale size $\theta_{c}$, detected by SPT at significance $\xi$, is given by

$$
\Delta \theta=\sqrt{\left(\theta_{\text {beam }}^{2}+\left(k \theta_{c}\right)^{2}\right)} / \xi
$$

where $\theta_{\text {beam }}$ is the beam FWHM, and $k$ is a factor of the order of unity (see Story et al. 2011 for more details). With this information, we estimate the expected cumulative distribution 
of the observed rBCG offsets, assuming a Gaussian with the appropriate width for each cluster; this is equivalent to assuming the underlying $\mathrm{rBCG}$ distribution to be a delta function centered at zero offset with respect to the true cluster SZ centroid. Results are shown as the blue dotted curve in Figure 7. It is clear that the observed distribution of $\mathrm{rBCG}$ offsets is broader than that expected if all rBCGs were located exactly at the cluster center. We conduct a K-S test to address the similarity of the two distributions. The hypothesis that the two distributions are drawn from the same parent distribution has a probability of $0.09 \%$, suggesting that the observed rBCG offset distribution cannot be easily explained by the SPT positional uncertainties alone.

Because the SPT candidate positional uncertainties $\Delta \theta$ are roughly the same, one can expect that our ability to measure the underlying $\mathrm{rBCG}$ distribution will weaken as we push to higher redshift where the cluster virial regions subtend smaller angles on the sky. We test this by dividing the sample into four redshift bins with similar numbers of members. The K-S tests confirm our expectations; using redshift bins of 0.0-0.40, $0.40-0.54,0.54-0.73$, and $z>0.73$, we find that the probability that the observed and positional error distributions are drawn from the same parent distribution is $0.11 \%, 0.008 \%, 1.97 \%$, and $43.4 \%$, respectively. Thus, with the current cluster sample, we cannot detect any extent in the rBCG distribution beyond a redshift $z \sim 0.7$. If we assume that the underlying $\mathrm{rBCG}$ offset distribution is Gaussian, the K-S test shows a maximum probability of $5.3 \%$ for a Gaussian distributed width of $0.074 r_{200}$ with the probability of consistency dropping below $0.1 \%$ for $\sigma>0.08 r_{200}$. Therefore, while the Gaussian is not a particularly good fit, the measured distribution strongly favors $\sigma<0.08 r_{200}$.

We test whether our SZ-selected cluster sample exhibits similar rBCG offsets to those seen in previous X-ray studies. To do this, we adopt the previously published BCG offset distribution from the X-ray studies as the underlying BCG offset distribution for our sample and then convolve this distribution with the SPT candidate positional uncertainties. If rBCGs in SZ-selected clusters are no different from those in these previously studied samples, then we would expect the K-S test probability of consistency to be high. We explore two samples: X-ray model 1 (Lin \& Mohr 2004; green dashed line in Figure 7) and X-ray model 2 (Mann \& Ebeling 2012; magenta dotted line in Figure 7). The probability of consistency between the SPT sample and X-ray model 1 is $41 \%$, and the probability of consistency between the SPT sample and X-ray model 2 is $0.46 \%$. We also examine another X-ray sample (Stott et al. 2012) which produces a very similar result with our X-ray model 2 with the probability of consistency of $0.55 \%$.

It appears likely that the differences between the two previously published X-ray samples can be explained in terms of differences in the BCG selection. The measured rBCG offset distribution presented in this work agrees with the Lin \& Mohr (2004) sample, in which the BCGs were defined as the brightest $K$-band galaxy projected within the virial radius $\theta_{200}$ with spectroscopic redshift consistent with the cluster redshift. This BCG selection is very similar to the SPT rBCG selection, with the main difference being that we do not have spectroscopic redshifts for all rBCG candidates in the SPT sample. The agreement between the SZ- and X-ray-selected samples in this case suggests that there are no strong differences between the merger fractions in these two cluster samples.

The Mann \& Ebeling (2012) BCG sample, in contrast, was assembled using bluer optical bands, which are more sensitive to the star formation history. In addition, in cases where a second concentration of galaxies was found within the projected virial region, the central galaxy of the galaxy concentration coincident with the X-ray emission peak was chosen as the BCG, regardless of whether it was brighter or not (A. W. Mann 2012, private communication). This selection criteria would make it difficult to identify significantly offset BCGs, which would be more likely to be present in merging systems. Similarly, the Stott et al. (2012) BCG sample was assembled using $i$-band data and a prior on the offset that excludes any offset greater than $500 \mathrm{kpc}$. Such a prior would also bias the measured distribution against large offsets due to ongoing merger activity.

The rBCG offset distribution measured in the SPT SZselected sample of clusters does not provide any compelling evidence that SZ-selected clusters differ in their merger rate as compared to X-ray-selected clusters. It will be possible to test this more precisely once we have the more accurate X-ray cluster centers with consistent rBCG selections. Currently, the X-ray properties of only 15 SPT SZ-selected clusters have been published (Andersson et al. 2011; Benson et al. 2011); however, over 100 additional SPT-selected clusters have been approved for observation in on-going programs with Chandra and $X M M-N e w t o n$. With those data in hand we will be able to measure the rBCG offset distribution over the full redshift range of SPT clusters, allowing us to probe for evolution in the merger rates with redshift.

\section{CONCLUSIONS}

The SPT-SZ survey has produced an approximately masslimited, redshift-independent sample of clusters. Approximately $80 \%$ of these clusters are newly discovered systems; the SPT survey has significantly increased the number of clusters discovered through the SZ effect and the number of massive clusters detected at high redshift. In this paper, we present optical/NIR properties of 224 galaxy cluster candidates selected from $720 \mathrm{deg}^{2}$ of the SPT survey that was completed in 2008 and 2009. The results presented here constitute the subset of the survey in which the optical/NIR follow-up is essentially complete.

With a dedicated pointed follow-up campaign using groundand space-based optical and NIR telescopes, we confirm 158 out of 224 SPT cluster candidates and measure their photometric redshifts. We show that $18 \%$ of the optically confirmed sample lies at $z>0.8$, the median redshift is $z=0.57$, and the highest-redshift cluster is at $z=1.35 \pm 0.07$. We have undertaken a cross-comparison among three different cluster redshift estimators to maximize the precision in the presented photometric redshifts. For each cluster, we combine the redshift estimates from the three methods, accounting for the covariance among the methods. Using 57 clusters with spectroscopic redshifts, we calibrate the photometric redshifts and uncertainties and demonstrate that our combining procedure provides a characteristic final cluster redshift accuracy of $\Delta z /(1+z)=0.017$.

For the 66 candidates without photometric redshift measurements, we calculate lower redshift limits. These limits are set by the depth of the optical/NIR imaging and the band combinations used. For nine of these candidates there is evidence from NIR data that the cluster is a high-redshift system, and that we simply need deeper NIR data to measure a photometric redshift.

Under the assumption that all 66 candidates without photometric redshift measurements are noise fluctuations, we estimate the purity of the SPT-selected cluster sample as a function of the SPT detection significance $\xi$. Results are in good agreement with expectations for sample purity, with no single unconfirmed 
system above $\xi=6,>95 \%$ purity above $\xi=5$, and $\sim 70 \%$ purity for $\xi>4.5$. By requiring an optical/NIR counterpart for each SPT candidate, the purity in the final cluster sample approaches $100 \%$ over the full $\xi>4.5$ sample. The purity of the SPT cluster sample simplifies its cosmological interpretation.

Next, we examine the measured rBCG offset from the SZ candidate positions to explore whether SZ-selected clusters exhibit similar levels of ongoing merging as X-ray-selected samples. We show that the characteristic offset between the rBCG and the candidate position is $\sim 0.5$. We examine the radial distribution of rBCG offsets as a function of scaled cluster radius $r / r_{200}$ and show that a model where we include scatter due to SPT positional uncertainties assuming all BCGs are at cluster centers has only a $0.09 \%$ chance of consistency with the observed distribution. That is, the observed distribution is broader than would be expected from SPT positional uncertainties alone. If we assume the rBCG offset distribution is Gaussian, the observations rule out a Gaussian width of $\sigma>0.08 r_{200}$; however, even with smaller width a Gaussian distribution is only marginally consistent with the data. When comparing the SPT rBCG distribution with an X-ray-selected cluster sample with a similar rBCG selection criteria (Lin \& Mohr 2004), the SPT- and X-ray-selected rBCG distributions are similar, suggesting that their merger rates are also similar. Comparisons to other X-ray-selected samples are complicated by differences in $\mathrm{rBCG}$ selection criteria. For example, comparing to Mann \& Ebeling (2012), which selects BCGs using bluer optical bands, we find a significantly less consistent rBCG distribution compared to SPT. We conclude that SZ- and X-ray-selected cluster samples show consistent rBCG distributions, and note that BCG selection criteria can have a significant effect in such comparisons.

With the full $2500 \mathrm{deg}^{2}$ SZ survey completed in 2011, we are now working to complete the confirmations and redshift measurements of the full cluster candidate sample. Scaling from this $720 \mathrm{deg}^{2}$ sample with effectively complete optical follow-up, we estimate that the full survey will produce $\sim 500$ confirmed clusters, with approximately 100 of them at $z>0.8$. This sample of clusters will enable an important next step in cluster cosmological studies as well as the first detailed glimpse of the high-redshift tail of young, massive clusters.

The South Pole Telescope program is supported by the National Science Foundation through grant ANT-0638937. Partial support is also provided by the NSF Physics Frontier Center grant PHY-0114422 to the Kavli Institute of Cosmological Physics at the University of Chicago, the Kavli Foundation, and the Gordon and Betty Moore Foundation. The Munich group acknowledges support from the Excellence Cluster Universe and the DFG research program TR33 The Dark Universe. Galaxy cluster research at Harvard is supported by NSF grant AST-1009012, and research at SAO is supported in part by NSF grants AST-1009649 and MRI-0723073. The McGill group acknowledges funding from the National Sciences and Engineering Research Council of Canada, Canada Research Chairs program, and the Canadian Institute for Advanced Research.

Optical imaging data from the Blanco $4 \mathrm{~m}$ at Cerro Tololo Interamerican Observatories (programs 2005B-0043, 2009B0400, 2010A-0441, 2010B-0598) and spectroscopic observations from VLT programs 086.A-0741 and 286.A-5021 and Gemini program GS-2009B-Q-16 were included in this work. Additional data were obtained with the $6.5 \mathrm{~m}$ Magellan Telescopes and the Swope telescope, which are located at the Las Campanas Observatory in Chile. This work is based in part on observations made with the Spitzer Space Telescope (PIDs 60099, 70053), which is operated by the Jet Propulsion Laboratory, California Institute of Technology, under a contract with NASA. Support for this work was provided by NASA through an award issued by JPL/Caltech. The Digitized Sky Surveys were produced at the Space Telescope Science Institute under U.S. Government grant NAG W-2166. The images of these surveys are based on photographic data obtained using the Oschin Schmidt Telescope on Palomar Mountain and the UK Schmidt Telescope. The plates were processed into the present compressed digital form with the permission of these institutions.

Facilities: Blanco (MOSAIC), Blanco (NEWFIRM), Gemini:South (GMOS), Magellan:Baade (IMACS), Magellan:Clay (LDSS3), SPT, Spitzer (IRAC), Swope, VLT:Antu (FORS2), WISE

\section{APPENDIX}

\section{NOTABLE CLUSTERS}

SPT-CL J0337-6207. This candidate is optically unconfirmed but has small NIR blank-field probability in at least one data set $\left(P_{\text {blank }}=0.5 \%\right.$ in WISE data-also, $P_{\text {blank }}=18.2 \%$ in NEWFIRM data-see Section 3.2 for details).

SPT-CL J0428-6049. This candidate is optically unconfirmed but has a high SPT significance $(\xi=5.1)$ and small NIR blank-field probability in at least one data set $\left(P_{\text {blank }}=0.5 \%\right.$ in WISE data; see Section 3.2 for details). Though $P_{\text {blank }}=69.0 \%$ in NEWFIRM data, there is clear visual evidence of an NIR overdensity in the NEWFIRM images, but at $\sim 40^{\prime \prime}$ from the SPT position. Such a large offset is heavily disfavored by the fitting procedure, such that the model that minimizes the overall $\chi^{2}$ for the NEWFIRM data is effectively a blank field. The position of the WISE overdensity is consistent with the SPT position.

SPT-CL J0458-5741. This cluster is listed as optically unconfirmed, but it is also listed in Table 2 of R12 as coincident with the low-redshift cluster ACO 3298 (at a separation of $77^{\prime \prime}$ ). We see a clear red-sequence overdensity in our Magellan/IMACS data at $z \simeq 0.2$, centered on the Abell cluster position. The bestfit SZ core radius for this candidate is 2.5, which implies an SPT positional uncertainty of $\sim 0.5$, in which case a $77^{\prime \prime}$ offset is only a $\sim 2 \sigma$ outlier. However, visual inspection of a lightly filtered SPT map shows that the SZ signal is coming from two distinct components, one of which corresponds to the Abell cluster position, and neither of which would have been significant enough to be included in the R12 catalog on its own. For this reason, we leave the $\theta_{c}=2.5$ candidate, which blends the SZ signal from the two individual components, as unconfirmed.

SPT-CL J2002-5335. This candidate is optically unconfirmed but has small NIR blank-field probability in at least one data set ( $P_{\text {blank }}=7.5 \%$ in WISE data-see Section 3.2 for details).

SPT-CL J2032-5627. This cluster is listed in Table 2 of R12 as coincident with the $z=0.06$ cluster $\mathrm{ClG} 2028.3-5637 / \mathrm{ACO}$ 3685 (at a separation of $115^{\prime \prime}$ from the literature Abell cluster position) and as coincident with the $z=0.14$ cluster RXC J2032.1-5627 (at a separation of 87" from the reported REFLEX cluster position). However, from our Magellan/IMACS imaging data, we estimate a red-sequence redshift of $z=0.31 \pm 0.02$, and, using the red-sequence measurements as a criterion for cluster member selection, we have obtained spectra for 32 cluster members using GISMO and have measured a robust spectroscopic redshift of $z_{\text {spec }}=0.2840$. Examination of the REFLEX spectroscopic catalog (Guzzo et al. 2009) reveals 
that their spectroscopic observations yielded five galaxies near their reported redshift of $z_{\text {spec }}=0.1380$ but also six galaxies within $2 \%$ of the value we derive from our GISMO observations $\left(z_{\mathrm{spec}}=0.2840\right)$. The value of $z_{\text {spec }}=0.0608$ for ACO 3685 is from only one galaxy (and, while reported in Struble \& Rood 1999, is originally from Fetisova 1981). We conclude that there are two clear optical overdensities at different redshifts along the line of sight to this SZ/X-ray system, and that the literature redshift of $z=0.0608$ for ACO 3685 is probably incorrect. Because of the redshift dependence of the SPT selection function (see, e.g., Vanderlinde et al. 2010), it is likely that the bulk of the SZ signal is coming from the higher-redshift cluster. We have obtained XMM-Newton data on this system, and the X-ray and SZ signals have very similar morphology, indicating that the $\mathrm{X}$-ray signal is also predominantly associated with the higher-redshift system. This makes it likely that the $z=0.2840$ system is a massive cluster and that the $z=0.1380$ system is a low-mass interloper, possibly the cluster originally identified as ClG 2028.3-5637/ACO 3685. SPT-CL J20325627 is discussed further-including weak-lensing data from Magellan/Megacam-in High et al. (2012).

SPT-CL J2035-5614. This candidate is optically unconfirmed but has small NIR blank-field probability in at least one data set ( $P_{\text {blank }}=0.1 \%$ in WISE data-see Section 3.2 for details).

SPT-CL J2039-5723. This candidate is optically unconfirmed but has a small SZ core radius (0.5) and small NIR blank-field probability in at least one data set $\left(P_{\text {blank }}=1.2 \%\right.$ in WISE data and $8.7 \%$ in NEWFIRM data-see Section 3.2 for details).

SPT-CL J2121-5546. This candidate is optically unconfirmed but has small NIR blank-field probability in at least one data set $\left(P_{\text {blank }}=0.9 \%\right.$ in WISE data-also, $P_{\text {blank }}=11.5 \%$ in NEWFIRM data-see Section 3.2 for details).

SPT-CL J2136-5535. This candidate is optically unconfirmed but has small NIR blank-field probability in at least one data set ( $P_{\text {blank }}=5.2 \%$ in WISE data-see Section 3.2 for details).

SPT-CL J2152-4629. This candidate is optically unconfirmed but has a high SPT significance $(\xi=5.6)$, a small SZ core radius $(0.25)$, and small NIR blank-field probability in at least one data set $\left(P_{\text {blank }}=8.0 \%\right.$ in WISE data; also $P_{\text {blank }}=10.6 \%$ in Spitzer/IRAC data and $20.0 \%$ in NEWFIRM data-see Section 3.2 for details). This is the only candidate with Spitzer/ IRAC $P_{\text {blank }}<20 \%$ for which we were not able to estimate a redshift.

SPT-CL J2343-5556. This candidate is optically unconfirmed but has small NIR blank-field probability in at least one data set $\left(P_{\text {blank }}=5.6 \%\right.$ in WISE data and $21.0 \%$ in NEWFIRM data-see Section 3.2 for details).

\section{REFERENCES}

Andersson, K., Benson, B. A., Ade, P. A. R., et al. 2011, ApJ, 738, 48 Appenzeller, I., Fricke, K., Fürtig, W., et al. 1998, Messenger, 94, 1 Ashby, M. L. N., Stern, D., Brodwin, M., et al. 2009, ApJ, 701, 428 Battye, R. A., \& Weller, J. 2003, Phys. Rev. D, 68, 083506

Benson, B. A., de Haan, T., Dudley, J. P., et al. 2011, arXiv:1112.5435

Bertin, E. 2006, in ASP Conf. Ser. 351, Astronomical Data Analysis Software and Systems XV, ed. C. Gabriel, C. Arviset, D. Ponz, \& S. Enrique (San Francisco, CA: ASP), 112

Bertin, E., \& Arnouts, S. 1996, A\&AS, 117, 393

Bertin, E., Mellier, Y., Radovich, M., et al. 2002, in ASP Conf. Ser. 281, Astronomical Data Analysis Software and Systems XI, ed. D. A. Bohlender, D. Durand, \& T. H. Handley (San Francisco, CA: ASP), 228

Blakeslee, J. P., Franx, M., Postman, M., et al. 2003, ApJ, 596, L143 Böhringer, H., Schuecker, P., Guzzo, L., et al. 2004, A\&A, 425, 367 Bower, R. G., Lucey, J. R., \& Ellis, R. S. 1992, MNRAS, 254, 601 Brodwin, M., Gonzalez, A. H., Moustakas, L. A., et al. 2007, ApJ, 671, L93
Brodwin, M., Gonzalez, A. H., Stanford, S. A., et al. 2012, ApJ, 753, 162 Brodwin, M., Ruel, J., Ade, P. A. R., et al. 2010, ApJ, 721, 90

Brott, I., \& Hauschildt, P. H. 2005, in The Three-Dimensional Universe with Gaia, ed. C. Turon, K. S. O’Flaherty, \& M. A. C. Perryman (ESA Special Publication, Vol. 576; Noordwijk: ESA), 565

Bruzual, G., \& Charlot, S. 2003, MNRAS, 344, 1000

Buckley-Geer, E. J., Lin, H., Drabek, E. R., et al. 2011, ApJ, 742, 48

Carlstrom, J. E., Ade, P. A. R., Aird, K. A., et al. 2011, PASP, 123, 568

Cease, H., Gosling, A. J., Doolin, S., et al. 2008, Proc. SPIE, 7014, 221

Collister, A. A., \& Lahav, O. 2004, PASP, 116, 345

Covey, K. R., Ivezić, Ž., Schlegel, D., et al. 2007, AJ, 134, 2398

Desai, S., Armstrong, R., Mohr, J. J., et al. 2012, ApJ, 757, 83

Eikenberry, S., Elston, R., Raines, S., et al. 2006, Proc. SPIE, 6269, 626917

Eisenhardt, P. R., De Propris, R., Gonzalez, A. H., et al. 2007, ApJS, 169, 225

Eisenhardt, P. R. M., Brodwin, M., Gonzalez, A. H., et al. 2008, ApJ, 684, 905

Fassbender, R., Böhringer, H., Nastasi, A., et al. 2011, New J. Phys., 13, 125014 Fazio, G. G., Hora, J. L., Allen, L. E., et al. 2004, ApJS, 154, 10

Fetisova, T. S. 1981, SvA, 25, 647

Finoguenov, A., Guzzo, L., Hasinger, G., et al. 2007, ApJS, 172, 182

Finoguenov, A., Watson, M. G., Tanaka, M., et al. 2010, MNRAS, 403, 2063

Foley, R. J., Andersson, K., Bazin, G., et al. 2011, ApJ, 731, 86

Garg, A., Stubbs, C. W., Challis, P., et al. 2007, AJ, 133, 403

Geller, M. J., \& Beers, T. C. 1982, PASP, 94, 421

Gladders, M. D., \& Yee, H. K. C. 2005, ApJS, 157, 1

Gonzalez, A. H., et al. 2010, BAAS, 216, 415.13

Guzzo, L., Schuecker, P., Böhringer, H., et al. 2009, A\&A, 499, 357

Haarsma, D. B., Leisman, L., Donahue, M., et al. 2010, ApJ, 713, 1037

Haiman, Z., Mohr, J. J., \& Holder, G. P. 2001, ApJ, 553, 545

High, F. W., Hoekstra, H., Leethochawalit, N., et al. 2012, ApJ, 758, 68

High, F. W., Stalder, B., Song, J., et al. 2010, ApJ, 723, 1736

High, F. W., Stubbs, C. W., Rest, A., Stalder, B., \& Challis, P. 2009, AJ, 138, 110

Holder, G., Haiman, Z., \& Mohr, J. J. 2001, ApJ, 560, L111

Hu, W. 2003, Phys. Rev. D, 67, 081304

Jannuzi, B. T., \& Dey, A. 1999, in ASP Conf. Ser. 191, Photometric Redshifts and the Detection of High Redshift Galaxies, ed. R. Weymann, L. StorrieLombardi, M. Sawicki, \& R. Brunner (San Francisco, CA: ASP), 111

Katayama, H., Hayashida, K., Takahara, F., \& Fujita, Y. 2003, ApJ, 585, 687 Kelson, D. D. 2003, PASP, 115, 688

Koester, B. P., McKay, T. A., Annis, J., et al. 2007, ApJ, 660, 239

Kurtz, M. J., \& Mink, D. J. 1998, PASP, 110, 934

Lima, M., \& Hu, W. 2005, Phys. Rev. D, 72, 043006

Lima, M., \& Hu, W. 2007, Phys. Rev. D, 76, 123013

Lin, Y.-T., \& Mohr, J. J. 2004, ApJ, 617, 879

Lloyd-Davies, E. J., Romer, A. K., Mehrtens, N., et al. 2011, MNRAS, 418, 14 Majumdar, S., \& Mohr, J. J. 2003, ApJ, 585, 603

Majumdar, S., \& Mohr, J. J. 2004, ApJ, 613, 41

Mancone, C. L., Gonzalez, A. H., Brodwin, M., et al. 2010, ApJ, 720, 284

Mann, A. W., \& Ebeling, H. 2012, MNRAS, 420, 2120

Mantz, A., Allen, S. W., Ebeling, H., \& Rapetti, D. 2008, MNRAS, 387, 1179

Mantz, A., Allen, S. W., Rapetti, D., \& Ebeling, H. 2010, MNRAS, 406, 1759

Marriage, T. A., Acquaviva, V., Ade, P. A. R., et al. 2011, ApJ, 737, 61

Melin, J.-B., Bartlett, J. G., \& Delabrouille, J. 2006, A\&A, 459, 341

Miknaitis, G., Pignata, G., Rest, A., et al. 2007, ApJ, 666, 674

Mohr, J. J., Adams, D., Barkhouse, W., et al. 2008, Proc. SPIE, 7016, 70160L

Molnar, S. M., Haiman, Z., Birkinshaw, M., \& Mushotzky, R. F. 2004, ApJ, 601,22

Muzzin, A., Wilson, G., Lacy, M., Yee, H. K. C., \& Stanford, S. A. 2008, ApJ, 686, 966

Muzzin, A., Wilson, G., Yee, H. K. C., et al. 2009, ApJ, 698, 1934

Ngeow, C., Mohr, J. J., Alam, T., et al. 2006, Proc. SPIE, 6270, 627023

Pacaud, F., Pierre, M., Adami, C., et al. 2007, MNRAS, 382, 1289

Papovich, C. 2008, ApJ, 676, 206

Perlmutter, S., Aldering, G., Goldhaber, G., et al. 1999, ApJ, 517, 565

Planck Collaboration, et al. 2011, A\&A, 536, A8

Reichardt, C. L., Stalder, B., Bleem, L. E., et al. 2012, arXiv:1203.5775

Rest, A., Stubbs, C., Becker, A. C., et al. 2005, ApJ, 634, 1103

Sanderson, A. J. R., Edge, A. C., \& Smith, G. P. 2009, MNRAS, 398, 1698

Sayers, J., Czakon, N. G., Bridge, C., et al. 2012, ApJ, 749, L15

Schmidt, B. P., Suntzeff, N. B., Phillips, M. M., et al. 1998, ApJ, 507, 46

Sifon, C., Menanteau, F., Hasselfield, M., et al. 2012, arXiv:1201.0991

Skrutskie, M. F., Cutri, R. M., Stiening, R., et al. 2006, AJ, 131, 1163

Song, J., Mohr, J. J., Barkhouse, W. A., Warren, M. S., \& Rude, C. 2012, ApJ, 747,58

Stalder, B., Ruel, J., Suhada, R., et al. 2012, ApJ, in press (arXiv:1205.6478)

Stanford, S. A., Brodwin, M., Gonzalez, A. H., et al. 2012, ApJ, 753, 164

Staniszewski, Z., Ade, P. A. R., Aird, K. A., et al. 2009, ApJ, 701, 32 
Stern, D., Eisenhardt, P., Gorjian, V., et al. 2005, ApJ, 631, 163

Story, K., Aird, K. A., Andersson, K., et al. 2011, ApJ, 735, L36

Stott, J. P., Collins, C. A., Sahlén, M., et al. 2010, ApJ, 718, 23

Stott, J. P., Hickox, R. C., Edge, A. C., et al. 2012, MNRAS, 422, 2213

Struble, M. F., \& Rood, H. J. 1999, ApJS, 125, 35

Šuhada, R., Song, J., Böhringer, H., et al. 2012, A\&A, 537, A39

Sunyaev, R. A., \& Zel'dovich, Y. B. 1972, Comments Astrophys. Space Phys., 4, 173

Tran, K.-V. H., Franx, M., Illingworth, G. D., et al. 2007, ApJ, 661, 750

Vanderlinde, K., Crawford, T. M., de Haan, T., et al. 2010, ApJ, 722, 1180
Vikhlinin, A., Kravtsov, A. V., Burenin, R. A., et al. 2009, ApJ, 692, 1060

Vikhlinin, A., McNamara, B. R., Forman, W., et al. 1998, ApJ, 502, 558

Wang, L., \& Steinhardt, P. J. 1998, ApJ, 508, 483

Wang, S., Khoury, J., Haiman, Z., \& May, M. 2004, Phys. Rev. D, 70,123008

Werner, M. W., Roellig, T. L., Low, F. J., et al. 2004, ApJS, 154, 1

White, S. D. M., Efstathiou, G., \& Frenk, C. S. 1993, MNRAS, 262, 1023

Williamson, R., Benson, B. A., High, F. W., et al. 2011, ApJ, 738, 139

Wright, E. L., Eisenhardt, P. R. M., Mainzer, A. K., et al. 2010, AJ, 140, 1868

Zenteno, A., Song, J., Desai, S., et al. 2011, ApJ, 734, 3 